\title{
CrystEngComm
}

Check for updates

Cite this: CrystEngComm, 2019, 21 3534

Received 9th January 2018 Accepted 24th April 2019

DOI: $10.1039 / \mathrm{c} 8 \mathrm{ce} 00042 \mathrm{e}$

rsc.li/crystengcomm

\section{Designs of continuous-flow pharmaceutical crystallizers: developments and practice}

\begin{abstract}
Mo Jiang (iD *ab and Richard D. Braatz (iD) ${ }^{a}$
Crystallization is an effective, low-cost purification \& formulation process widely applied to pharmaceuticals and fine chemicals. This review describes recent advances in research on lab-scale solution-based continuous crystallization, including (1) a 5-step general design procedure; (2) key design/operational parameters; (3) process intensification strategies; and (4) a case study. The continuous crystallizers reviewed include mixed-suspension mixed-product removal, fluidized beds, oscillatory baffled flow, and tubular laminar/segmented/slug-flow crystallizers. Their corresponding design and operational considerations are summarized in terms of general parameters (e.g., residence time), and crystallizer-specific parameters and strategies (e.g., mixing strategies). In-line nucleation and crystal modification methods are categorized, including use of micromixers, wet milling, ultrasonication, temperature cycling, and recycling selection (filtration, sedimentation). Throughout the article, links are drawn with extensive existing knowledge of batch crystallizers, to facilitate the understanding and design of continuous crystallizers.
\end{abstract}

\section{Introduction}

Recent years have witnessed rapidly increased research from both academia and industry on continuous solution crystallization for reproducible and inexpensive manufacturing of high-quality products. ${ }^{1-11}$ Continuous crystallization contributes to high product reproducibility, high labor efficiency, and low capital and production costs, ${ }^{9-11}$ with applications such as high-volume precursors in polymer manufacturing, ${ }^{12,13}$ compression tableting, and inhalers. ${ }^{6}$ Continuous crystallizers suit for solute-solvent(s) systems of fast nucleation and growth kinetics. ${ }^{14}$ This review focuses primarily on recent advances of continuous crystallizers (for solution-based crystallization), including process design and parameters, generally applicable process intensification strategies, and practical insights.

Successful designs of continuous crystallization process are almost always based on good understanding of batch processes. For example, most existing crystallizations in the pharmaceutical industry are batch or semibatch, ${ }^{4,5,15,16}$ with significant existing expertise and knowledge on equipment design, process monitoring/control, and training of operating personnel. Batch crystallizers also handle process startup

\footnotetext{
${ }^{a}$ Department of Chemical Engineering, Massachusetts Institute of Technology, 77 Massachusetts Avenue, E19-551, Cambridge, MA, 02139, USA

${ }^{b}$ Department of Chemical and Life Science Engineering, Virginia Commonwealth University, Richmond, VA, 23219, USA. E-mail: mjiang3@vcu.edu; Tel: +1 8048274001
}

and control better, with flexible seeding choice, and less potential of clogging or fouling. Batch crystallizers are suited for slow to medium nucleation and growth kinetics. This article also discusses how to combine the strengths of both batch and continuous crystallizers, in section 2 (steps 2 and 3 ), sections 3 and 4 (the development of several continuous crystallizers and seeding methods), section 5 (the choice of crystallizer configuration for the case study), and section 6 (outlook).

Section 2 describes a 5-step design procedure (Fig. 1a) for continuous crystallizers based on the authors' own experience: (1) design the product specifications (e.g., solid forms) and process requirements (e.g., yield, residence time); (2) carry out batch crystallization experiments to obtain preliminary values of thermodynamic and kinetic parameters in step 1 ; (3) select the operational mode (batch or continuous) based on the requirements and characteristics in steps 1 and 2; (4) select the continuous crystallizer configuration based on steps 1-3 (e.g., stirred tank, column, or tube geometry, details in section 3), together with configuration-specific parameters (Table 1); and (5) design and improve the details for the selected crystallizer configuration, including seeding strategies (section 4) and all parameter values in steps 1-4. The steps 13 are applicable to the design of any crystallizer, whereas steps 4 and 5 are focused on continuous designs (with the same underlying principles applicable to both batch and continuous crystallization). As with any other guide, this approach serves as a starting point for suitable design and selection, and aims to inspire continuous improvement and new procedures. 
(a)

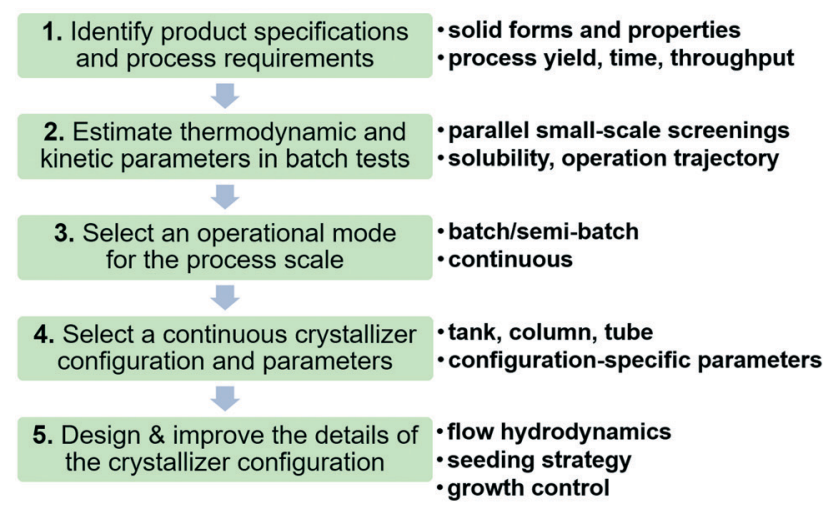

(b)

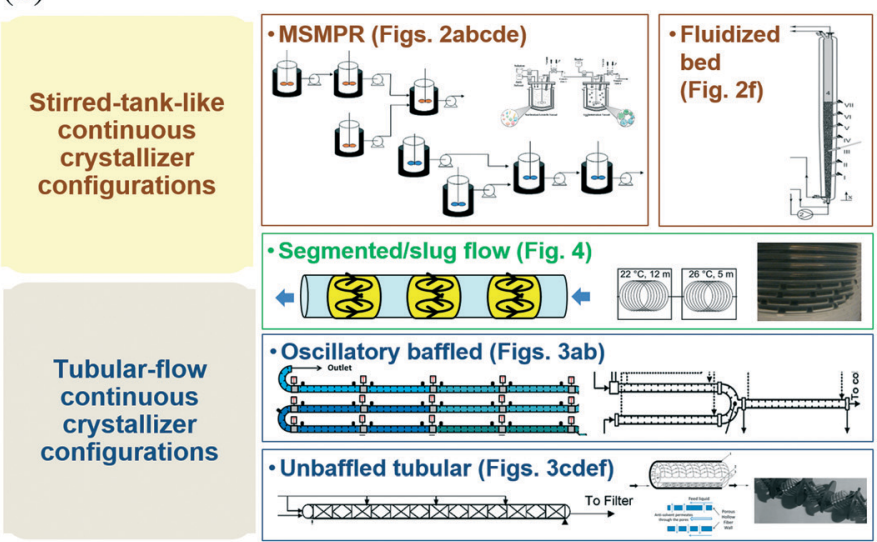

Fig. 1 (a) Five steps (green box, justified in section 1) and corresponding key considerations (black bulleted lists) for continuous crystallizer design. The crystallizer design and improvement (steps 4 and 5) are further elaborated in sections 2 to 5. (b) Stirred-tank and tubular-flow categorization of continuous crystallizer configurations. Due to the page limit, only partial images of representative configurations are listed and shown here. The full images of each configuration are detailed in later figures (figure numbers labelled). Modified with permission from ref. 10, 14, 29, 41, 43, 53, 82 and 86, Copyright 2010, 2011, 2012, 2014, 2015 American Chemical Society, ref. 7, 30, 34, 35, 46, 72 and 77, with permission from Elsevier, and ref. 17 and 108, with permission from Wiley, respectively. Key qualities for these crystallizer configurations are compared in Table 1, with parameters summarized.

Table 1 Properties and parameters of continuous crystallizers based on key process/product requirements. The advantages general to the crystallizer geometry are discussed in detail in step 4 of section 2, thus not repeated here, such as existing knowledge for stirred tanks, and improved heat transfer for tubular geometry. Representative crystallizer images and parameters are listed here, not meant to be comprehensive. The detailed analysis of general and configuration-specific parameters are in section 2 (steps 1-3) and section 3 (corresponding crystallizer configurations and their mixing strategies), respectively. The specific values of residence time and flow rate for many individual papers are reviewed elsewhere, ${ }^{68}$ thus not repeated here

\begin{tabular}{|c|c|c|c|c|c|}
\hline $\begin{array}{l}\text { Continuous } \\
\text { crystallizers }\end{array}$ & MSMPRs & $\begin{array}{l}\text { Fluidized bed } \\
\text { crystallizers }\end{array}$ & $\begin{array}{l}\text { Oscillatory baffled } \\
\text { crystallizer }\end{array}$ & $\begin{array}{l}\text { Unbaffled tubular flow } \\
\text { crystallizers }\end{array}$ & $\begin{array}{l}\text { Slug/segmented flow } \\
\text { crystallizers }\end{array}$ \\
\hline Crystallizer geometry & Tank & $\begin{array}{l}\text { Conical } \\
\text { shape tube }\end{array}$ & Baffled column/pipe & Tube & Tube \\
\hline
\end{tabular}

Mixing strategy

Mechanical stirrer

Fig. 2a-e

Example process and/or equipment images

Residence time examples

$\begin{array}{ll}\text { Residence } & \text { Feed/seed } \\ \text { time }^{65}{ }^{65} \text { flow } & \text { flow rate } \\ \text { rate }^{53} & \end{array}$

Inlet slurry

flow from

Oscillatory fl
baffled pipe

bottom up

(anded pipe

Fig. $2 f$

Fig. 3a and $b$

Recirculation Oscillation amplitude,

Configuration-specific Stage parameters
Feed/seed flow rate, ${ }^{40,43}$ oscillatory Reynolds number ${ }^{40,43}$ volume ${ }^{50,60}$ recycle collection ratio $^{50,59}$ stirrer height $^{46}$ agitation rate ${ }^{53}$ (shear rate)
Intrinsic recirculation within slugs rotating cylinder, membran hollow fiber), or coil tubing

Fig. $3 c-f$

Fig. 4

\section{Oscillation amplitude, Crystallizer/reactor} baffle number, ${ }^{40}$ baffle spacing, open cross-sectional area, ${ }^{43}$ oscillatory frequency ${ }^{40}$
Residence time, ${ }^{9,80,81}$ total flow rate $^{41,79,84,85}$

temperature, ${ }^{81}$ anti-solvent injection points, ${ }^{41}$ cylinder rotation speed, ${ }^{82,83}$ membrane pore size $^{84,85}$ flow inverter designs ${ }^{91}$
Residence time, ${ }^{9,95}$ flow rate (or tubing length)

Slug size, ${ }^{10}$ segmenter configuration, ${ }^{17}$ in-line seeding (e.g., sonication amplitude, ${ }^{14}$ micromixer configuration $^{10}$ )
Section 3 reviews the main classes of lab-scale continuous crystallizer configurations (Table 1 and Fig. 1b) studied since the last decade, with mixing strategies and key parameters categorized (Table 1). These crystallizers include: (a) multistage MSMPR crystallizers; (b) fluidized bed crystallizers; (c) baffled tubular crystallizers (aka oscillatory flow crystallizer); (d) unbaffled tubular crystallizers, with mixing enhancers (e.g., static mixers) inside or coiled tube; and (e) segmented/ slug flow crystallizers. While many of the tubular continuous crystallizers were called "plug flow crystallizer" in existing literature, this review distinguishes them based on key design and physical features (e.g., flow or mixing). In addition, comparison and description are included for those continuous crystallizers with a batch mode (e.g., baffled tubular crystallizers) or batch-similar equipment (e.g., MSMPRs). The corresponding design and operational variables for all continuous 
crystallizers are summarized into general parameters (residence time) and crystallizer-specific parameters and mixing strategies. The parameters listed in Table 1 are mainly for residence time (flow rate) and mixing, both of which directly affect kinetics, with detailed analysis in section 3 on corresponding crystallizer configurations. The parameters that affecting process yield (solubility-based) are also important, such as the temperature and concentration sets, ${ }^{10}$ solvent to anti-solvent ratio, or reactant ratio. ${ }^{17}$ But these parameters have been well reviewed in crystallization textbooks/literature and discussed in step 2 of section 2, and not listed specifically in Table 1.

Section 4 focuses on seeding strategies in continuous flow. As with batch crystallizers, seeding can reduce the maximum supersaturation level needed for nucleation ${ }^{18}$ and facilitate better product quality (e.g., higher purity and less aggregation). Based on existing practice, the strategies are categorized for seed generation, addition, and (optional) treatment, with two modes each: discrete (off-line) and continuous (in $s i t u$ ). Five in-line methods are elaborated, with principles and design/operation parameters in Table 2: micromixers, wet milling, ultrasonication, temperature cycling, and recycling selection method (filtration, sedimentation). All methods for the continuous mode can be used in an off-line or batch mode. The direct feedback control of nucleation in micro- scale flow can be more challenging than in batch crystallizers: the smaller volume makes the crystallizer less feasible for sensor installation. Also, while low supersaturation can evidently increase nucleation induction time, high supersaturation may promote undesired nucleation and even aggregation (and clogging). A more realistic goal is to be able to tune nucleation rate and crystal sizes in flow, through which to indirectly control the crystal number density and size distribution. Multiple in-line methods can also be used simultaneously on the same continuous crystallizer, such as in the case study in the next section.

Section 5 is a case study that further describes the procedure and strategies in sections 2-4, using a proof-of-concept millifluidic design for continuous cooling crystallization of a model compound, with multiple in-line methods for focused nucleation and crystal treatment. To avoid repetition with existing case studies of well-developed reactors/crystallizers and/or model compounds (e.g., in section 3 and references thereof), this case study is on cooling crystallization of an organic molecule, L-asparagine monohydrate (LAM), in gas-liquid segments/slugs at mesoscale. The development and application of segmented/slug flow in process crystallization are currently at an early stage, but the physical phenomena have been quickly recognized and adopted in microfluidic reactions, and increasingly in larger scales, to control quality of

Table 2 Common in-line methods for generating small crystals in continuous flow, from solution (in situ nucleation) and/or slurry (in-line crystal treatment). The size reduction and increase of crystals are indicated by - and +, respectively. Representative equipment is listed here, not including pumps and tubing that are generally needed for most continuous crystallizers. Example parameters and applicable crystallizers for these in-line methods are listed, not meant to be comprehensive. Here "tubular crystallizers" refer to both laminar-flow and segmented/slug flow crystallizers. Further details of these methods are in section 4

\begin{tabular}{|c|c|c|c|c|c|}
\hline $\begin{array}{l}\text { In-line } \\
\text { methods }\end{array}$ & Micromixers & Ultrasonication & $\begin{array}{l}\text { High-shear wet } \\
\text { milling }\end{array}$ & T cycling & $\begin{array}{l}\text { Recycling selection } \\
\text { method }\end{array}$ \\
\hline $\begin{array}{l}\text { Physical } \\
\text { principle }\end{array}$ & $\begin{array}{l}\text { Momentum of mixing } \\
\text { streams }^{8,28}\end{array}$ & $\begin{array}{l}\text { Accelerate nucleation } \\
\text { and crystal breakage }\end{array}$ & $\begin{array}{l}\text { High shear for } \\
\text { crystal fracture } \\
\text { and nucleation }\end{array}$ & $\begin{array}{l}\text { Temperature-dependent } \\
\text { solubility }\end{array}$ & $\begin{array}{l}\text { Size-dependent } \\
\text { permeability or } \\
\text { sedimentation }\end{array}$ \\
\hline $\begin{array}{l}\text { Crystal size } \\
\text { change }\end{array}$ & + & $-1-$ & $+1-$ & $+/-$ & $+/-$ \\
\hline
\end{tabular}

Representative Micromixers

lab equipment

Equipment Jet/vortex/grid mixers configurations (Fig. 4c and 5a-c)

Parameter examples

Continuous crystallizer examples and 4)

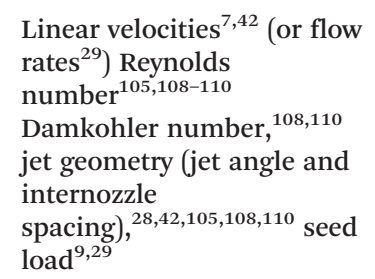

Sonicators

Sonication probe

(Fig. 6), sonication bath (Fig. 4b)

Tubular crystallizers (Fig. 3
Amplitude (energy, intensity), ${ }^{14,114,120,140,141}$ sonic exposure time $\mathrm{i}^{12}$ (flow rate), frequency ${ }^{13}$

Tubular crystallizers (Fig. 4), fluidized bed (Fig. 2f)
Rotor stator mixers

Mill head ${ }^{124}$ (Fig. 7) or tooth geometry (coarse, medium, fine, multi-stage)

Tip/rotational speed, ${ }^{54,122,124}$ turn over (or cycle) number and/per residence time ${ }^{55,124,126}$

MSMPR (Fig. 2c) All (Fig. 2-4) units
Temperature control Filters, columns

Crystallizer jacket (Fig. 2a and b), circulation bath (Fig. 4b and c), heat exchanger (Fig. 3a and b)

Residence time, ${ }^{10,104} \mathrm{~T}$ range and ramp, ${ }^{10,104} \mathrm{~T}$ cycle number ${ }^{104}$
Filters (Fig. 2d) (glass filter, ${ }^{64}$ filter paper, membranes), column (Fig. 2e)

Recycle ratio, ${ }^{63,64}$ MSMPR recycle stage, flow rate, filter properties $^{59}$ (MW cut off, hydrophobicity), seed size ${ }^{46}$
MSMPR (Fig. 2c-e), fluidized bed (Fig. 2f) 
nanoparticles and/or nanocrystals. ${ }^{19-26}$ The slug flow tubular crystallizer also maintains useful operational properties of both batch (e.g., less clogging) and continuous crystallizers (e.g., scale $\mathrm{up}^{27}$ ), and improves crystal qualities in flow. Nucleation and growth subprocesses are uniquely controlled, by generating a localized region with high supersaturation, while reducing the overall supersaturation for the whole crystallizer. ${ }^{28}$ The solution is only in contact with tubing to minimize contamination. ${ }^{28}$ Detailed design process with key experimental details and pitfalls are included to accelerate further improvement and optimization. Besides LAM, other model compounds were also demonstrated in cited references for slug flow crystallizers, such as acetylsalicylic acid ${ }^{29}$ and succinic acid. ${ }^{30}$

Section 6 provides an outlook on the design of continuous crystallizers, including some ideas on where the field is probably going, how the process requirement is going to change, and other helpful elements such as process intensification strategies and process control.

\section{A 5-step approach for designing continuous crystallizers}

Step 1 is to set up goals, based on the product specifications and process requirements (Fig. 1a). Typical goals ${ }^{4,5}$ include achieving target solid forms and/or crystal properties ${ }^{6,8,14,31,32}$ (e.g., molecular purity, polymorph, crystal size distribution, ${ }^{14}$ morphology) at a desired process outcome (e.g., yield, residence time, production rate). The goals for crystallization can vary at different stages of manufacturing. For example, for the crystallization of pharmaceutical intermediates, the main specifications are tight control of molecular purity and yield, ${ }^{6}$ with moderate specifications on the crystal size and shape distribution (only need to ensure efficient washing and filtration), as the crystals are usually re-dissolved in subsequent downstream processing. For the final crystallization of a purified drug substance, ${ }^{6}$ the control of crystal size, shape, and solid form becomes more important. Reasons include that the crystal size and shape affects dosage uniformity, tablet stability, and bioavailability, and the specific polymorphic form affects the solubility and hence the bioavailability. ${ }^{33}$

Step 2 is to collect thermodynamic and kinetic data. A large number of parallel small-scale screening experiments should be carried out to select the solvent(s), the compound composi$\operatorname{tion}^{31}$ (e.g., single component or cocrystal ${ }^{34}$ ), and the specific polymorphic form. ${ }^{35}$ These screening experiments enable the exploration of a wide range of solvents, solvent mixtures, evaporation rates, cooling rates, and $\mathrm{pH}$ shifts. Although such data collection can be carried out in continuous flow, ${ }^{5}$ batch screening experiments are much more efficient in materials use. $^{36,37}$ The data from these screening experiments should be reviewed to identify any potential processing issues such as aggregation, solvent inclusions, or undesired crystal shapes. ${ }^{33}$ Detailed process technology for solid form selection and generation has been well reviewed. $5,15,35$
Then the solubility is better quantified, again usually in batch crystallizers. ${ }^{38}$ The solubility profile is used to choose the crystallization methods, including cooling, evaporation, anti-solvent ("drowning-out"), $\mathrm{pH}$ shift, or reaction ("precipitation") crystallizations. For example, cooling crystallization requires strong temperature-dependence of solubility. Anti-solvent, $\mathrm{pH}$ shift, and reaction have to consider possible non-ideal mixing of solutions of different compositions. Sometimes several methods are combined (e.g., cooling followed by anti-solvent or evaporation), usually for the pur-

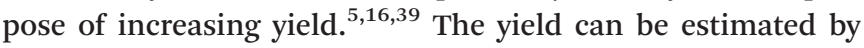
the solubility difference between starting and ending conditions in the crystallizer. Note that solubility is affected by the impurity profile in the solvent, which at the early stage can be very different from the final manufacturing process. ${ }^{33}$

Step 3 is to select the operational mode for the process scale, that is, batch/semi-batch or continuous. The preferred operational mode depends on the goals in step 1 - especially on purity, yield, and throughput - together with operational considerations. The advantages for batch and continuous crystallizers have been compared in section 1 . The former has been thoroughly reviewed elsewhere, ${ }^{4,5,15,16}$ and the latter is described in this article such as in step 4. Many times business decisions play an important role, such as deadlines and/ or staff recruitment/training time.

Crystal properties such as solid forms and purity largely depend on the operational trajectory on the crystallization phase diagram. The operational trajectory indirectly specifies the time or spatial profile of supersaturation, by specifying the time or spatial profile of temperature (using tanks ${ }^{10}$ or water jackets $^{40}$ ), solvent/anti-solvent ratio, ${ }^{41}$ and/or the amount of solvent evaporated. ${ }^{4,16}$ Operational trajectories of low supersaturation (e.g., the solution concentration near the solubility curve) tend to produce crystals of the highest molecular purity. Operational trajectories of high supersaturation can be allowed when the molecular impurities have a low tendency to be incorporated into the crystals. High supersaturation not only promotes nucleation and growth, ${ }^{4,16}$ but also possible agglomeration, ${ }^{31}$ or even the formulation of a liquid-liquid dispersion. ${ }^{39}$ The maximum theoretical yield of a continuous crystallization process is the same as a batch process with the same beginning and end conditions (e.g., temperature and/or composition, and concentration). Usually it is desired to maximize the yield, while maintaining acceptable crystal characteristics ${ }^{42}$ within the operation limit, such as preventing undesired solvent evaporation and boiling, ${ }^{42}$ and compound decomposition at high temperatures. A poorly designed continuous crystallizer can reduce the yield, such as by fouling (e.g., early precipitation) on the interior walls of tubular crystallizer or having the solution leaving the crystallizer still being supersaturated.

As with any technology, continuous crystallizers have their own intrinsic challenges to solve. For example, if the main objective was to control the crystal size distribution, then ideal tubular plug flow with a uniform velocity profile is desired, ${ }^{43}$ due to its consistent hydrodynamics and enhanced 
heat/mass transfer from strong radial mixing and negligible axial mixing, and a nonzero velocity close to the wall reduces the potential for fouling and clogging. In reality, the velocity profile in a tube/pipe is parabolic, which can result in a very wide residence time distribution: ${ }^{44}$ some crystals in the tube center quickly move through the tube and are smaller, whereas some crystals near the walls tend to stay in the tube for very long times and grow, and/or even foul or clog the tube. Tubular crystallizers investigated in recent years have been designed to approach ideal plug flow while being practically implementable, as reviewed in section 3.

Step 4 is to select a continuous crystallizer configuration and corresponding parameters. Crystallizers in research laboratories (Table 1) can be roughly placed within one of two categories (Fig. 1b): mixed tanks and plug-flow-like tubular crystallizers. General advantages of these crystallizer are summarized here, with descriptions and parameters detailed in section 3 for further information.

Mixed-tank crystallizers have the advantages of (i) longer residence time and higher spatially averaged crystal area, which allows operation at lower supersaturation and so higher molecular purity, and (ii) easier to reconfigure existing batch/semibatch crystallizer equipment, at least for some designs. The use of multiple stages allows the supersaturation to be nearly zero at the exit of the last mixed tank, which maximizes the yield. The simplest mixed-tank design, known as mixed-suspension mixed-product removal (MSMPR, Fig. 2a-e), uses an appropriate designed mixer to provide sufficient mixing that the slurry is nearly spatially homogeneous in each tank, so that slurry that continuously leaves each tank has the same crystal size distribution as the spatially averaged CSD. MSMPRs are simple crystallizers to build and model. An alternative mixed-tank design is the fluidized bed crystallizer (Fig. 2f), which uses an uplifting fluid flow to suspend the crystals and generate mixing. The lack of a mixing blade reduces fluid shear and crystal breakage, ${ }^{45,46}$ and the extent of mixing can be tuned by varying the aspect ratio of the bed and the rate in which liquid is recycled through the column. The fluidized bed crystallizer can be designed so that the larger crystals can be preferentially removed, which results in a narrower product crystal size distribution than achievable using an MSMPR.

The plug-flow-like tubular crystallizers have the advantages of (i) narrower residence time distribution and increased uniformity and control of the crystal size distribution, (ii) improved external heat transfer ${ }^{43}$ and temperature control due to large surface area/volume ratio, and (iii) lower shear. Among these crystallizers, oscillatory baffled crystallizers (Fig. 3a and b) approximate many stirred-tank in series and allow for the longest residence time and flexible flow/mixing patterns and operation modes (periodic motion of baffles or fluid). ${ }^{43}$ Tubular crystallizers with internal mixing enhancers (e.g., static mixers, a rotating cylinder, membrane fibers) or coiled packing is a simpler approach that creates radial mixing without requiring any external moving parts (Fig. 3c-f). Segmented/slug-flow crystallizer
(Fig. 4) is a configuration that benefits from the existing experience and advantages of both batch and continuous crystallizers, such as low potential for clogging when designed and operated properly (e.g., have no constrictions), and easy to set up with low cost.

Step 5 is to design the details of the selected crystallizer configuration. One common parameter for all continuousflow crystallizers is the flow hydrodynamics (or mixing). For any specific crystallizer geometry, the flow rate not only needs to be designed before experiments, but also can serve as an intrinsic degree of freedom to facilitate quality control. For example, a high flow rate in a tubular crystallizer improves mixing through enhanced heat and mass transfer, for more uniform temperature and concentrations spatially for crystallization phenomena. But if the flow rate is too high, then the residence time can be too short, which leads to possibly lower yield. Other typical design considerations are to avoid unexpected or uncontrolled temperature variations, ${ }^{47}$ and to take into account the heat of mixing and solvent separation for anti-solvent crystallization. Key parameters for specific crystallizer configurations (include mixers) are listed in Table 1, with details in section 3 . The details for nucleation in continuous flow are elaborated in section 4 .

\section{Continuous crystallizer configurations and their key design parameters}

\section{Multi-stage MSMPRs}

Multi-stage MSMPRs have been used to produce crystals of high molecular purity ${ }^{48,49}$ at high yield, and to modify crystal properties, mostly in cooling and anti-solvent crystallizations (Fig. 2ac and e). ${ }^{4,6,50}$ The industrial version of MSMPRs are referred to as forced circulation crystallizers. ${ }^{4,5,16}$ In each MSMPR, feed solution/slurry is continuously pumped into a stirrer-agitated vessel, ${ }^{5}$ with product slurry nominally being continuously withdrawn at the same composition as in the vessel. ${ }^{50-57}$ Usually multiple MSMPRs are used to maximize the overall yield. Single-stage MSMPR has also been investigated in research studies: (1) the single-stage yield and/or purity has been improved by combining with continuous recycling of mother liquor or crystals after treatment ${ }^{58-64}$ (details in seeding strategy section and Table 2), (2) the polymorphic ratio has been controlled by varying the temperature and residence time, ${ }^{65}$ and (3) singlestage MSMPR experiments have been used to provide information for the design of multi-stage MSMPRs. ${ }^{60,65}$ Compared to multi-stage MSMPRs, the single-stage MSMPR crystallizer ${ }^{59}$ has lower running cost, whereas multi-stage MSMPRs have narrower residence time distributions, ${ }^{43,63}$ and less tendency towards fouling/clogging when the transfer line is cleared. ${ }^{66}$ The equipment for MSMPRs are very similar to standard stirred batch crystallizers, with continuous flow for inlet and outlet, thus shorter average residence time within each vessel (which can be compensated by increasing the number of vessels and/or residence time per container ${ }^{6}$ ). 
(a)

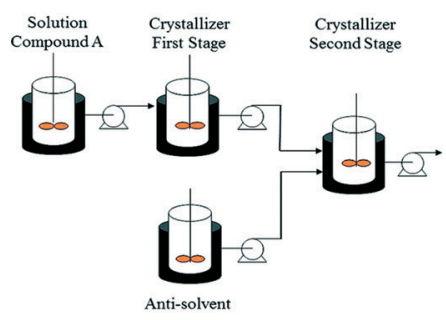

Configuration A

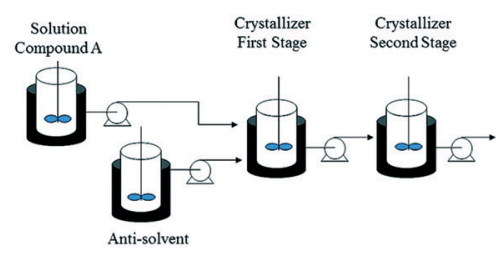

Configuration B

(c)
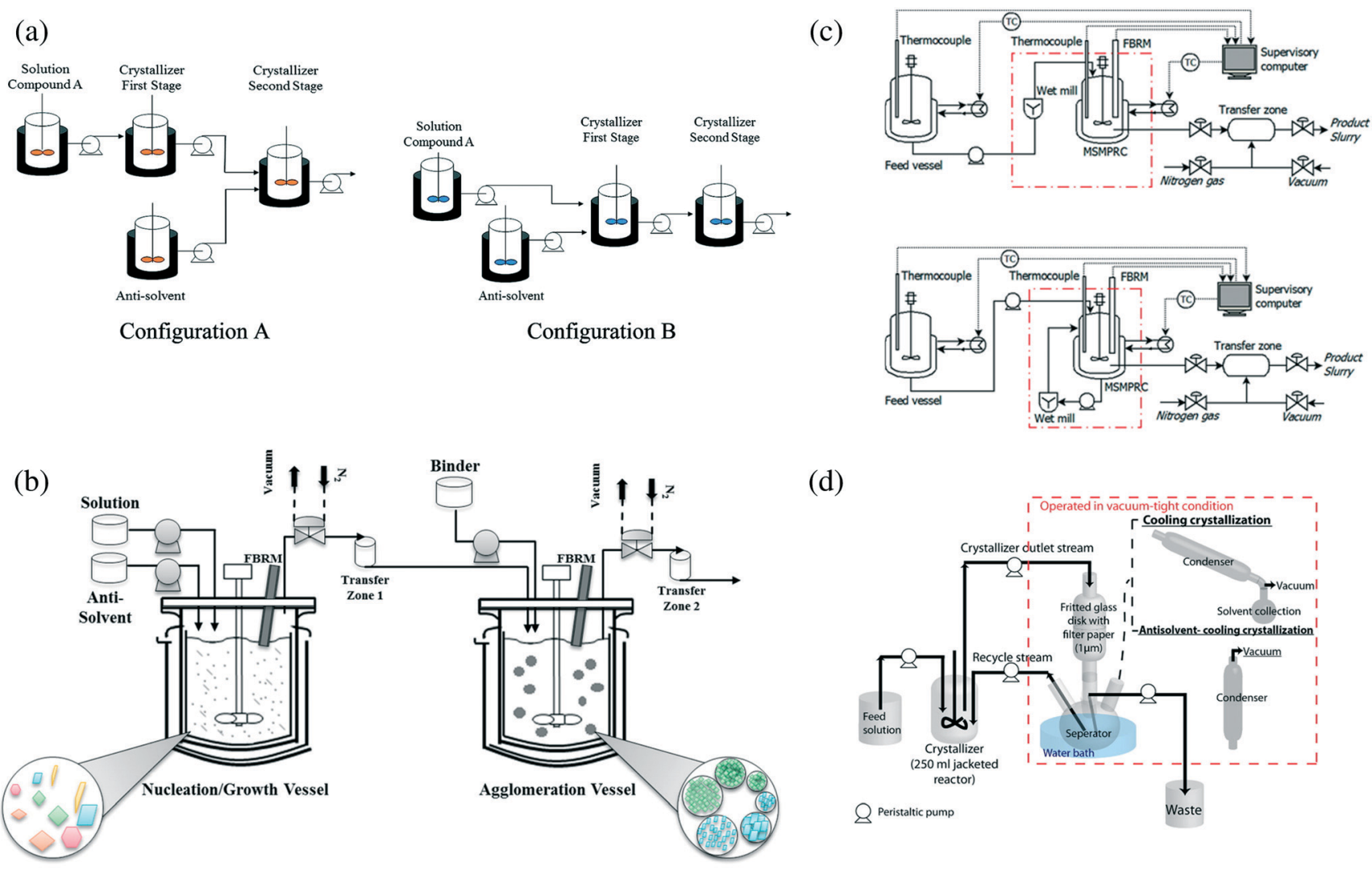

(d)

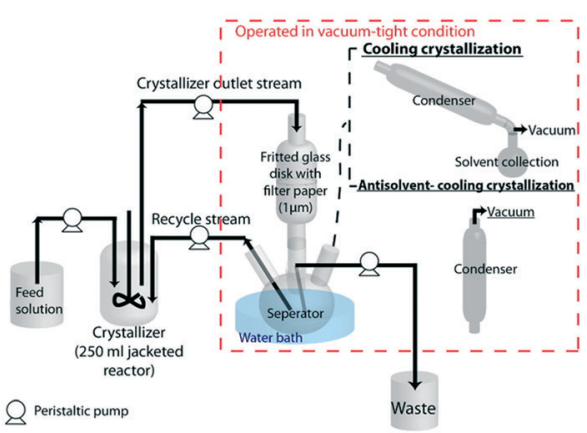

(e)

(f)
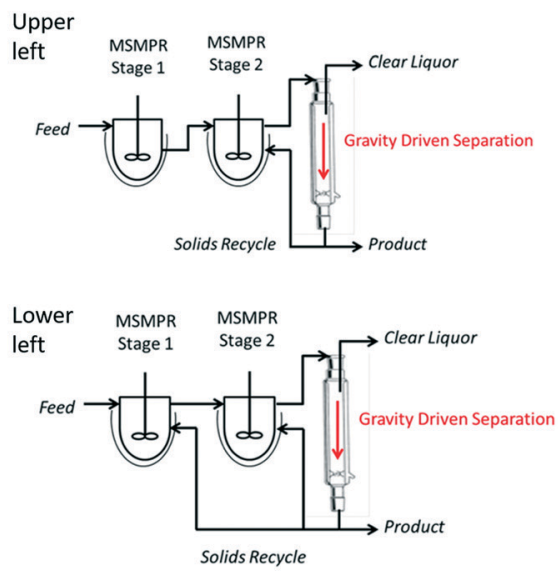

Right
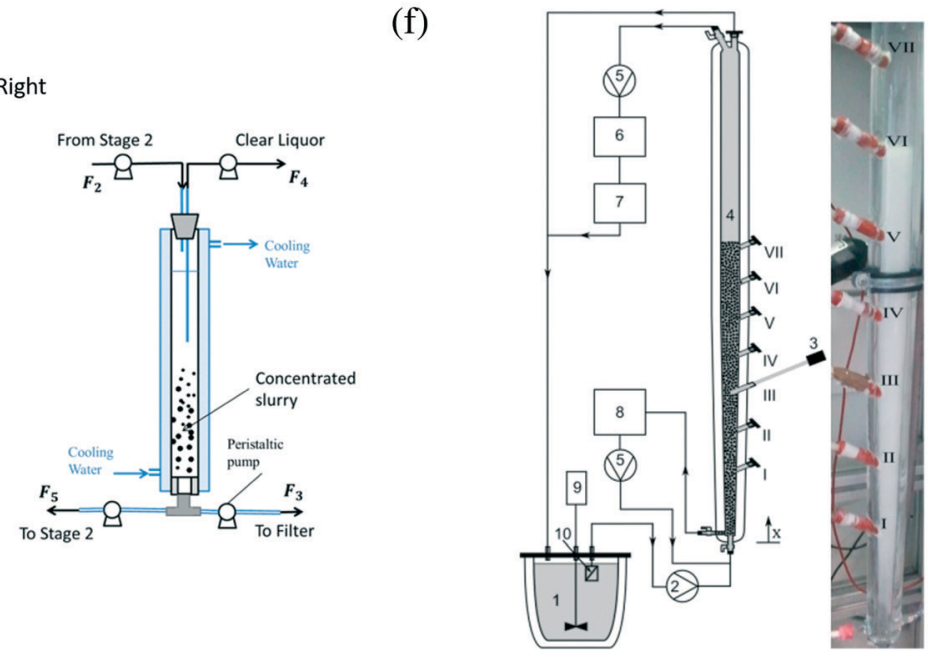

Fig. 2 (a) Two configurations of two-stage MSMPR that allow cooling, anti-solvent, and combined crystallization. ${ }^{6}$ All vessels have controlled temperature for cooling. Reprinted with permission from ref. 6, Copyright 2012 American Chemical Society. (b) Two-stage MSMPR combining antisolvent crystallization and spherical agglomeration. ${ }^{53}$ Reprinted with permission from ref. 53, Copyright 2015 American Chemical Society. (c) Twostage MSMPR with wet milling for (upper) nucleation and for (lower) crystal size reduction. ${ }^{55}$ Reprinted with permission from ref. 55 , Copyright 2015 American Chemical Society. (d) Single-stage MSMPR with a filtration recycling loop. ${ }^{64}$ Reprinted with permission from ref. 64 , Copyright 2012 American Chemical Society. (e) Two-stage MSMPR with solids recycling to (upper left) the second stage and (lower left) both stages; (Right) column separator for selecting solids from mother liquor. ${ }^{63}$ Reprinted with permission from ref. 63, Copyright 2016 American Chemical Society. In (a) to (e), each stage of the MSMPR setup can be very similar to a batch crystallizer (e.g., a jacketed crystallizer with an overhead stirrer ${ }^{6}$ and process analytical technology ${ }^{53}$ ) except for the continuous inlet and outlet flows. (f) Diagram (left) and photo (right) of a fluidized bed crystallizer for isomeric separation with cooling crystallization. ${ }^{46}$ Saturated solution is continuously drawn from the feed tank to the bottom of the crystallizer, where part of slurry is drawn and comminuted in an ultrasonication bath before being sent back. ${ }^{8,46}$ Part of the solution exits at the top of the crystallizer back into the feed tank. ${ }^{8,46}$ Reprinted from ref. 46 , with permission from Elsevier.

The agitation rate is set based on the purpose of the specific stage. Low to moderate agitation rate is used to limit sec- ondary nucleation and reduce aggregation. ${ }^{4,53}$ The higher the residence time (e.g., from using a larger operating volume), 
(a)

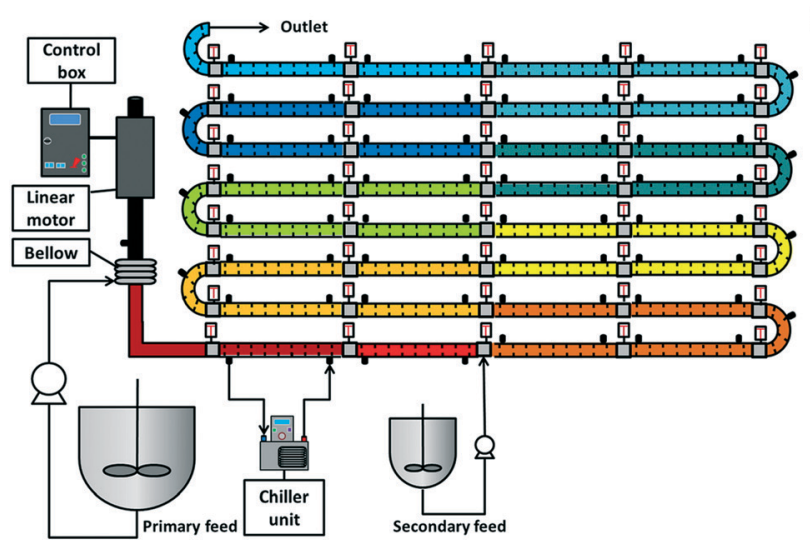

(c)

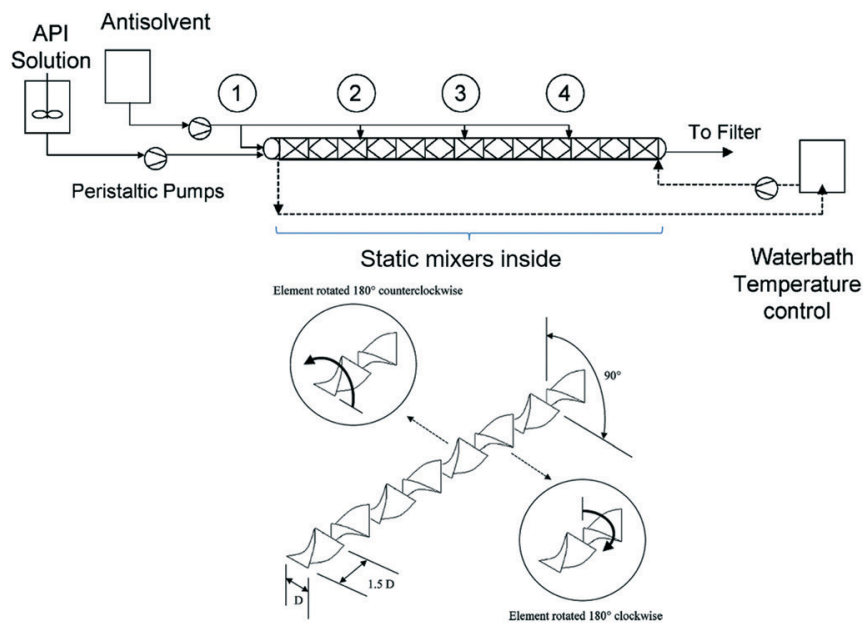

(b)

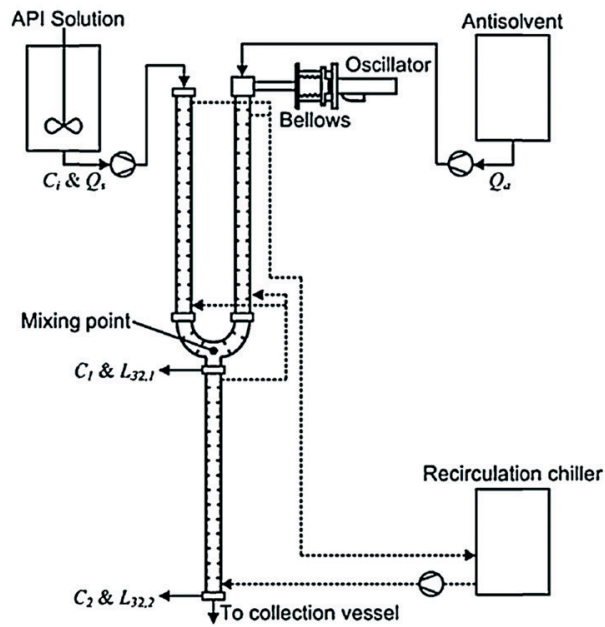

(d)

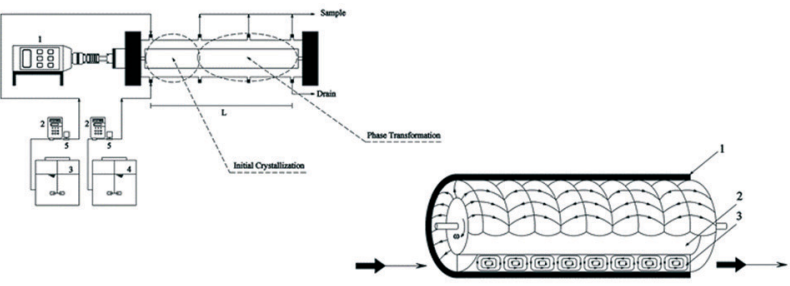

(e)

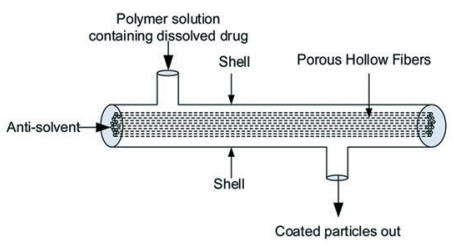

(f)

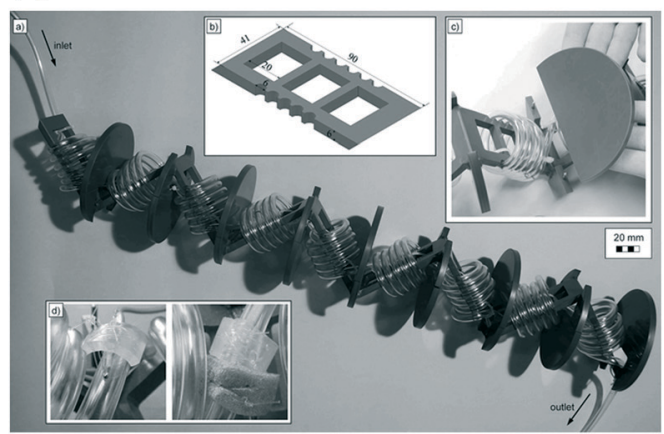

Fig. 3 (a) Continuous oscillatory baffled crystallizer for cooling crystallization (with chiller and temperature control units) and co-crystallization. ${ }^{43}$ Reprinted with permission from ref. 43, Copyright 2015 American Chemical Society. (b) Continuous oscillatory baffled crystallizer for anti-solvent crystallization..$^{72}$ In both (a) and (b), baffles were placed periodically along the length of the crystallizer include bends. ${ }^{40}$ Reprinted from ref. 72 , with permission from Elsevier. (c) Continuous crystallizer with static mixers inside. ${ }^{41}$ The numbers 1 to 4 indicate the injection points of antisolvent. The mixers (e.g., Kenics type) split and guide the total flow (solution and antisolvent) to homogenize the fluid. ${ }^{41}$ Reprinted with permission from ref. 41, Copyright 2010 American Chemical Society. (d) Continuous Couette-Taylor crystallizer. ${ }^{82}$ The Taylor vortices are generated by the rotating inner cylinder inside the stationary outer cylinder. Reprinted with permission from ref. 82, Copyright 2011 American Chemical Society. (e) Continuous crystallizer with membrane hollow fibers inside. ${ }^{86}$ Antisolvent permeate through the membrane pores and mix with the solution stream. Reprinted with permission from ref. 86, Copyright 2014 American Chemical Society. (f) Continuous tubular crystallizer with coiled flow inverters. ${ }^{79,91}$ The complete setup includes multiple plates, with each plate (containing coiled tube and connection) shown in the upper right-hand side. ${ }^{79}$ Reprinted from ref. 79 , with permission from Wiley. 
the more time for crystal nucleation/growth (higher yield ${ }^{6}$ ) and aggregation maturation in the vessel. ${ }^{53}$ Simulation models based on population balance and solute and solvent mass balances $^{51,67}$ (possibly including an energy balance ${ }^{60}$ ) indicate that: (1) the higher the temperature or the lower the volume of the final stage, the lower the yield and the higher the purity of final product crystals. ${ }^{6,50}$ (2) the higher the recycle ratio, the higher the yield (and the lower the purity). ${ }^{50}$ Startup modes are also suggested to quickly achieve steady state and reduce fouling, including beginning with the crystallizer filled with solution or slurry, instead of a dry crystallizer; ${ }^{68}$ higher temperature in the feed line; ${ }^{12}$ and adopting dynamic operational profile and process control strategies. ${ }^{50-57}$

\section{Fluidized bed crystallizers}

Fluidized bed crystallizers (FBCs, Fig. 2f) have been used to make large crystals, ${ }^{45,46}$ and/or for isomeric separation, ${ }^{46,48,69}$ mainly via cooling crystallization. ${ }^{4,5,15}$ The Oslo crystallizer is an industrial version of an FBC, which works best for fragile organic crystals such as amino acids. ${ }^{16}$ In a typical FBC (e.g., Fig. 2f), supersaturated solution (with seeds) enters at the bottom of the crystallizer, and this upward liquid flow agitates the crystals in the crystallizer to form a fluidized bed. ${ }^{15}$ Liquid solution with depleted supersaturation exits the FBC at the top. ${ }^{15}$ The seeds are generated by ultrasonication breakage of larger crystals, at or near the bottom of the crystallizer. The conical shape of the crystallizer allows larger crystals to drop to the bottom, ${ }^{46}$ where they can be removed from the vessel.

The lower the height for sample collection within the same crystallizer, the larger the crystal size. ${ }^{46}$ The recirculation velocity (or feed flow rate) has upper and lower bounds, due to requirement of internal agitation, thermal exchange, and avoiding high supersaturation that would promote encrustation. ${ }^{16}$ Within the operation range, the larger average particle size at a higher feed flow rate has been explained by larger particle interactions (aggregation). ${ }^{46}$

\section{Oscillatory baffled crystallizers}

Oscillatory baffled crystallizers (OBCs, or "oscillatory/pulsed flow crystallizers", Fig. 3a and b) have been used for cooling crystallization, ${ }^{18,40,70,71}$ co-crystallization, ${ }^{43}$ and anti-solvent crystallization. ${ }^{72}$ OBCs enhance mixing while having low average forward flow rates by inserting periodically spaced baffles that cause turbulent eddies upon interaction with the oscillatory flow in the pipe. ${ }^{40}$ OBCs can run in either batch or continuous mode. The term "continuous" here refers to the continuous flow both into and out of the crystallizer, not the oscillatory motion of the pistons. Batch OBCs have no flow in/out, and their applications have been thoroughly reviewed elsewhere. ${ }^{43,73,74}$

In a continuous $\mathrm{OBC}$, higher agitation generates more radial mixing ${ }^{73}$ from a combined effect of larger oscillation amplitude and/or number of baffles, generates smaller crystals, and reduces aggregation. ${ }^{40}$ Other operational parameters (Table 1) have been evaluated in batch OBCs, including baffle designs (e.g., baffle shape ${ }^{74}$ spacing, open cross-sectional area ${ }^{43}$ ) and oscillation amplitude and frequency, ${ }^{40}$ which all af- fect flow patterns thus mixing intensities and eddy propagation. ${ }^{73}$ The effect of these parameters can be quantified by the oscillatory Reynolds number to characterize mixing intensity and the Strouhal number (the ratio of column diameter to amplitude) to characterize effective eddy propagation. ${ }^{43,73,74}$

\section{Unbaffled tubular crystallizers}

Continuous laminar flow in an unbaffled tubular crystallizer is a simple and common environment for various kinds of crystallization. However, based on fluid mechanics, the solvent/slurry velocity near the tubing wall ${ }^{75}$ is very small, which favors fouling and clogging. ${ }^{9,76,77}$ The spatial heterogeneity in the velocity, with the maximum velocity approximately twice the average velocity, also widens the residence time distribution. ${ }^{44}$ To reduce those potential issues, various design approaches (besides process control $^{78}$ ) have been implemented that change the flow pattern and increase mixing intensity: $:^{9,41,79,80}$

(1) Static mixers ${ }^{44,81}$ can be placed inside the tube (Fig. 3c), to enhance the mixing of solution and antisolvent ${ }^{41}$ or precipitants ${ }^{80}$ to keep particles in suspension and to narrow the crystal size distribution. ${ }^{41}$ For antisolvent crystallization, the number of antisolvent injection points ${ }^{41}$ which directly impacts the supersaturation profile and the total flow rate $^{41,80}$ which directly impacts the mixing intensity and residence time affect the final crystal size distribution, with the extent depending on the specific solute/solvent system. ${ }^{41}$

(2) A rotational cylinder can be placed inside a stationary cylinder to generate periodic Taylor vortices between the two cylinder surfaces for enhanced radial mixing (Fig. 3d). ${ }^{82,83}$ Crystallization occurs in the vortices, and the temperatures of both cylinder surfaces in contact with the vortices are controllable. The higher the rotation speed, the larger the energy dissipation and mass transfer coefficient, and the smaller the crystal size. ${ }^{82,83}$

(3) Membranes (porous hollow fiber) can also be placed inside a tube to continuously facilitate nanocrystal generation from antisolvent crystallization (Fig. 3e).$^{84-87}$ Among different designs, fouling can be reduced by placing the membranepermeable antisolvent in the tube (non-permeable solution in the shell), so that crystallization (and the mixing zone ${ }^{87}$ ) occurs at the shell side, with multiple inlet antisolvent flows through pores. ${ }^{84,85}$ The membrane pore size is an important factor for mixing and crystallization.

(4) Even without any inside structure addition, the tubing packing itself can be changed, from a straight line to coils ${ }^{88,89}$ or helical coils, ${ }^{9,90}$ to narrow residence time distribution, with possible further mixing enhancement from coiled flow inverters. ${ }^{79,91,92}$ Again the residence time (flow rate) directly affects the final crystal mean size. ${ }^{9}$ There are also design parameters belonging to the coiled flow inverters, such as coil diameter and number of bends. $^{79,91}$ The coil geometry (e.g., Fig. 3f) has also been used in advanced segmented/slug-flow crystallizers discussed below.

\section{Segmented/slug flow tubular crystallizers}

A narrow residence time distribution approaching plug flow can also be achieved by segmented/slug flow tubular 
crystallization. ${ }^{10,14,17,26,29,93-96}$ Each liquid segment/slug is a functional crystallizer itself, with mixing from recirculation (intrinsic properties of slug flow) without requiring mixing blades. ${ }^{9,10,14,29,97}$ Reactive precipitation, ${ }^{17,26,30,93-96}$ cooling crystallization, ${ }^{10,14,29,98,99}$ and anti-solvent crystallization, ${ }^{100}$ have all been demonstrated for milli-fluidic flow systems (that is, the tubing inner diameter and slug size is on the scale of millimeters), with residence time on the order of minutes or longer, depending on the production purposes. Alternating segments/slugs of slurry and a non-miscible phase (liquid or gas) have been formed using segmenters ${ }^{17,94,101,102}$ (aka flow cutters) or simple tubing fittings ${ }^{9,10,14,29,97}$ (e.g., Fig. 4b and c).

The sizes of these segments/slugs can be tuned to improve the recirculation flow pattern (e.g., Fig. 4d), and the crystal size distribution within each slug. ${ }^{10}$ For example, the small volume allows uniform spatial distribution of temperature and concentration within each slug, and a slug with an aspect ratio close to 1 also favors uniform distribution of crystals inside. ${ }^{10}$ The shorter the residence time, ${ }^{95}$ and/or the larger the seed load, the smaller the mean size increase of the product crystals. ${ }^{29}$ The scale-up for the slug flow crystallizer can be achieved without increasing the tubing diameter nor length: (1) by simply running the experiment for a longer time to generate more slugs; ${ }^{10,14}$ and/or (2) by lining up parallel tubular crystallizers at the same operating conditions. ${ }^{94}$ Using a process with residence time of 6 minutes as an example, scaling up 10 times the total production means running the crystallization process continuously for 60 minutes, at the same operational conditions (e.g., residence time, temperature profile) and in the same equipment. A case study for the slug-flow process with design strategies are discussed in section 5 .

\section{Seeding strategies for continuous flow}

\subsection{Seed generation}

The correct chemical (e.g., enantiomer ${ }^{103}$ ) and solid forms are selected before deciding on seed crystal size and loading, as described in step 1 of section 2. Most in situ seed generation methods can be applied off line.

(1) Off-line seed generation

The seed crystals come from a previous process (e.g., batch) and are treated (e.g., milling or sieving) before seeding continuously to the current process. Most current continuous crystallizer configurations have adopted this strategy, including the first stage of MSMPRs, oscillatory baffled crystallizers, and some fluidized bed crystallizers. ${ }^{69}$ All existing crystallizer configurations can be used to generate crystals as off-line seeds, especially varieties of batch crystallizer with controlled crystal polymorph, size, and morphology. The treatment of those off-line seeds for designed seed size are discussed in the off-line seed treatment in a later subsection.

(2) In situ seed generation

$\dagger$ Readers interested in micromixers in smaller scale microchannels/microreactors are directed elsewhere ${ }^{19,20}$ this article is focused on micromixers that are large enough to be practically used for crystallization at the manufacturing scale.

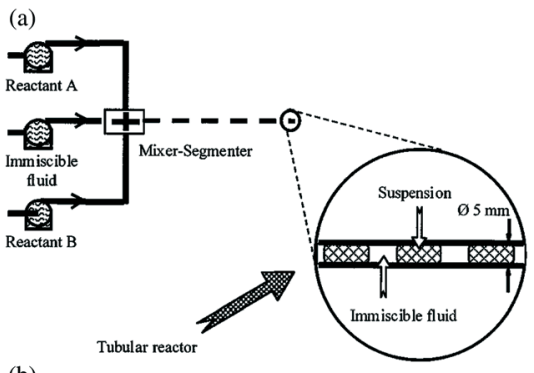

(b)

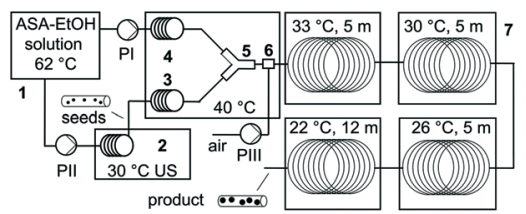

(c)

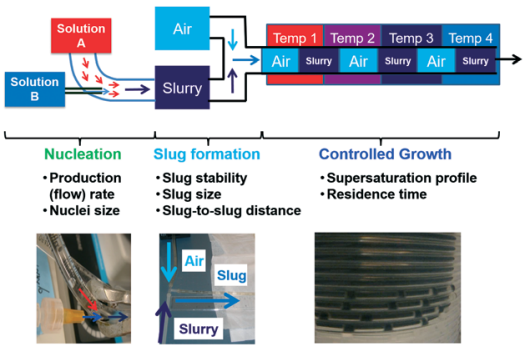

(d)

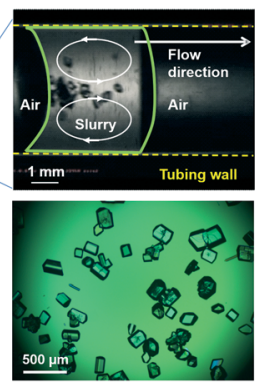

Fig. 4 (a) Segmented-flow crystallizer for reaction precipitation, with segments generated by a segmenter. ${ }^{17}$ Reprinted from ref. 17, with permission from Wiley. (b) Segmented/slug-flow crystallizer for cooling crystallization, ${ }^{29}$ with slugs generated by air inlet. A sonication bath is used for generating seeds, which are then mixed with saturated solution at a high temperature before slug formation. Reprinted with permission from ref. 29, Copyright 2012 American Chemical Society. (c) A slug-flow continuous crystallizer design with decoupled sub-processes (crystal nucleation, slug formation, and crystal growth). Each subprocess has the photos of corresponding key equipment shown in the bottom part of the figure, and the key design considerations listed then elaborated in section 2. For example, stable slugs in the slug-formation sub-process refers to slugs that do not combine nor separate after formation, which affects the recirculation mixing pattern within slugs and the control of crystal size distribution inside the slugs. The supersaturation could be generated from cooling, antisolvent, and/or reaction crystallization. Although the nucleation method shown in the figure uses micromixers, other methods can be used (e.g., ultrasonication in Fig. 6). Modified with permission from ref. 10, Copyright 2014 American Chemical Society. (d) (upper right) In-line video snapshot of a representative liquid slug (boundaries in green, internal fluid motion in white arrows), separated by two air slugs inside a tube (inner walls in yellow). ${ }^{134}$ The whole crystallizer is composed of a series of identical liquid slugs where crystallization occurs. Modified with permission from ref. 10, Copyright 2014 American Chemical Society. (Bottom) Offline microscope image of product crystals from a representative slug. Experimental conditions are detailed in ref. 10. 
The seed crystals can be continuously generated in the flow. This strategy has been adopted by segmented/slug flow crystallizers, ${ }^{10,14,27,104}$ laminar flow unbaffled crystallizers, ${ }^{41}$ micromixer crystallizers, multi-stage MSMPRs, and fluidized bed crystallizers. ${ }^{46}$ For example, in tubular crystallizer with an internal mixer inside tubing, antisolvent can be injected at various ports along tubing to continuously induce nucleation and growth. ${ }^{41}$ In multi-stage MSMPRs, upstream tanks (usually require off-line seed generation) can serve as in situ seed generators for downstream tanks. Three continuous seeding methods that originated from batch experiments are reviewed below.

(a)
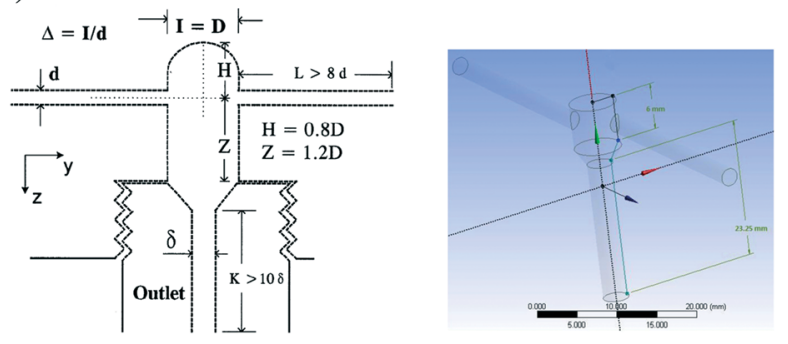

(b)
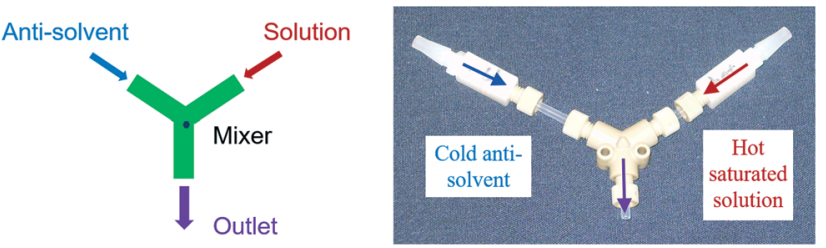

(c)
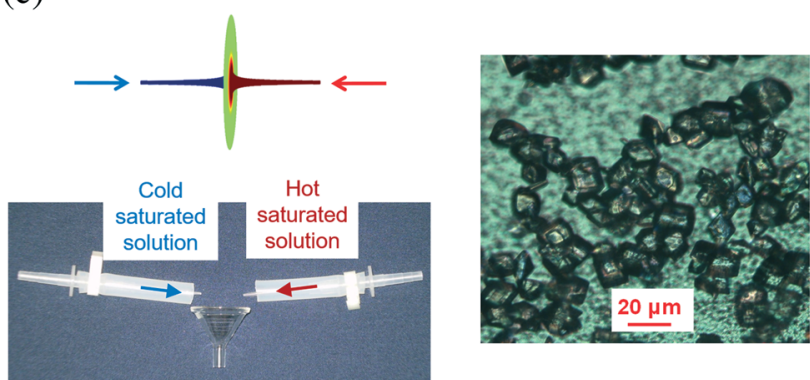

Fig. 5 (a) Diagrams of (left) confined submerged dual-impinging-jet (DIJ) micromixers; ${ }^{108}$ and (right) a vortex mixer. ${ }^{77}$ The centerlines for the two inlet jets align for DIJ, and are not aligned for vortex mixers. Reprinted from ref. 77 and 108, with permission from Wiley and Elsevier, respectively. (b) Diagram (left) and photo (right) of confined Y-shaped DIJ micromixers. ${ }^{7}$ Two jets collide onto each other for rapid mixing. The jet velocity is adjustable by varying the inner diameters of micromixers. ${ }^{7}$ Modified from ref. 7, with permission from Elsevier. (a) and (b) are for anti-solvent and reaction crystallizations. (c) Diagram and photo (left) of representative DIJ micromixers for cooling crystallization and the crystals produced (right).,28,47 Saturated solutions of different temperatures impinge onto each other from two opposed jets. Due to the flow symmetry, the impingement plane is in a circular shape. ${ }^{28}$ The tiny black dots on the background are the pores (size 2 $\mu \mathrm{m}$ ) of the membrane filters where crystals were filtered. ${ }^{47}$ Modified from ref. 30, 34 and 35, with permission from Elsevier.
Micromixers $\dagger$ have been used to generate seeds for batch, semi-batch, and tubular continuous crystallizers (e.g., laminar flow and segmented/slug flow crystallizers). Sometimes micromixers are called $\mathrm{T}$ mixer, $\mathrm{Y}$ mixer, or impinging jet mixers as compared in the next paragraph. Through rapid micromixing, homogeneous composition of supersaturated solution was created, for generating small nuclei in flow, ${ }^{42,105,106}$ mainly from anti-solvent ${ }^{7,42,107}$ (possibly combined with cooling) or reaction ${ }^{42,105,108}$ crystallizations. Recently cooling micromixers were developed, which continuously generate uniform crystals (Fig. 5c) by mixing two solutions of the same solvent at different temperatures and concentrations, under laminar flow. ${ }^{10,47}$ Unlike anti-solvent micromixers, complete composition mixing is not necessary or undesired for primary nucleation in cooling micromixers. ${ }^{28}$ The reason is that supersaturation for primary nucleation $^{28}$ was generated from the difference in kinetics between thermal mixing (faster) and composition mixing (slower). In most micromixers, the solution streams are only in contact mainly with tubing, which minimizes the potential for contamination.

There are multiple types of micromixers used for production scale (e.g., Fig. 5a-c), including impinging jet mixers ${ }^{7,42,105,108}$ and vortex mixers. ${ }^{77,109}$ Impinging jet mixers can be submerged or not submerged, ${ }^{108} \mathrm{~T}$ or $\mathrm{Y}$ shaped, ${ }^{7}$ sometimes with grids inside the mixing chamber to induce turbulence. ${ }^{105}$ Vortex mixers (Fig. 5a) allow unequal volumetric flow rates from inlets. For a specific micromixer
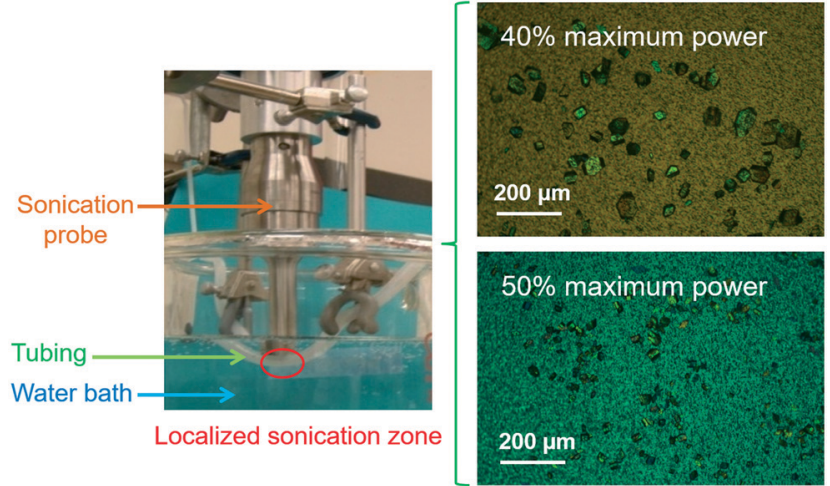

Fig. 6 (left) The focused indirect ultrasonication ${ }^{14}$ setup for flow nucleation, and microscope (with polarizers) images of crystals produced from the setup at $40 \%$ (upper right) and $50 \%$ (lower right) sonication amplitudes, respectively, without changing the flow rate nor other conditions. The ultrasonic probe is placed tightly against the outer wall of the silicone tubing inside which the solution flows, to generate a small nucleation zone ${ }^{118}$ (red ellipsoid) right below the probe tip. The heat generated from ultrasonication is removed by water in the water bath whose temperature was kept constant. ${ }^{14}$ The tiny black dots on the background are the pores of the membrane filters where nuclei were filtered. ${ }^{14}$ Reprinted with permission from ref. 14, Copyright 2015 American Chemical Society. Other sonication horns (e.g., flow-through ultrasonic horn ${ }^{115}$ ) or conventional use of a regular sonication probe (in direct contact with the solution) in interesting systems (e.g., near the jet outlet ${ }^{140}$ or in the side stream ${ }^{33}$ ) are elaborated in detail in the cited references. 
configuration, a higher jet velocity leads to higher mixing intensity, which affects the reaction product conversion $^{105,108,109}$ and particle size distribution, ${ }^{7}$ provided that the mixing time is slower than nucleation induction time (Damkohler number > 1). ${ }^{105}$ The larger the Damkohler number (relatively slower mixing compared to nucleation), the larger the mean particle size. ${ }^{110}$

Ultrasonication has been applied to batch, tubular laminar flow, and segmented/slug flow crystallizers for

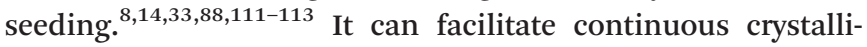
zation by accelerating nucleation rate ${ }^{29,70}$ (with reduced induction time and metastable zone width ${ }^{114}$ ), and generate micron-size crystals ( $c f$. Fig. 6$)^{8,14,33}$ or nanocrystals ${ }^{115}$ (e.g., spraying fine droplets into an anti-solvent stream). Although the exact physical mechanisms for aiding crystallization are not fully known, cavitation (microbubble growth and collapse ${ }^{116}$ ) is believed to play a crucial role. ${ }^{114}$ Most sonication parameters have been evaluated in batch crystallizers, such as the solution composition, sonication exposure time $^{12}$ frequency, and sonication intensity/energy (also studied in continuous crystallizers, as described in the next paragraph).
There are two main types of ultrasonication equipment for continuous crystallization: (1) baths ${ }^{15,17,29,46}$ are convenient to use, and can prevent direct contact of metal equipment with the solution, which lower the potential for contamination and sample thermal degradation, and (2) probes $^{12,115,117}$ allow higher and adjustable intensity with more options of probe shapes. Various configurations have been built and designed based on each of these equipment types, including focused indirect sonication ${ }^{14,118}$ (Fig. 6) that combines the advantages of both indirect contact and adjustable intensity within a small focused zone. Within a reasonable range, ${ }^{119}$ the higher the sonication amplitude (intensity/ energy), the smaller the induction time ${ }^{120}$ and crystal $\operatorname{size}^{14}$ (e.g., as in Fig. 6). Recent studies on the direct consequence of ultrasonication into liquid, which are pressure waves, showed that the effective sonication intensity received by the samples is spatially heterogeneous, ${ }^{13,118}$ thus the placement of ultrasound source (e.g., a probe, or the transducer(s) in a sonication bath) with respect to the samples have a strong effect on the nucleation outcome.
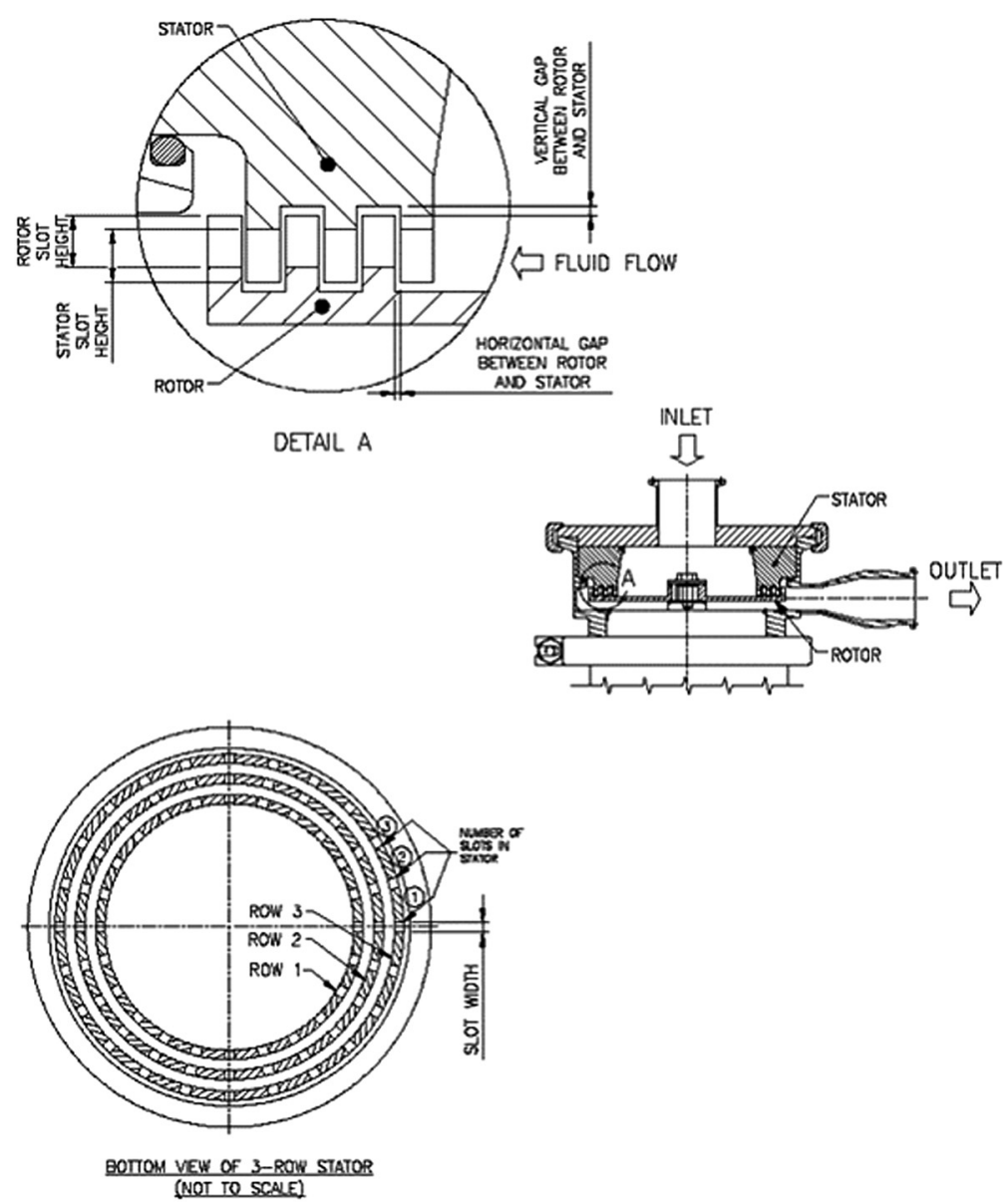

Fig. 7 Schematic view of the tooth geometry of a typical rotor-stator mill. ${ }^{124}$ The slots in the rotor and stator and their heights can vary. Reprinted with permission from ref. 124, Copyright 2013 American Chemical Society. 
Although mainly used for reducing crystal size, high-shear wet milling has also been applied to MSMPR crystallizers for in-line nucleation from solution (Fig. 2c), with fast kinetics for process control. ${ }^{54-57,121}$ Primary and secondary nucleation are accelerated when the solution goes through the wet mill with high shear. ${ }^{54-57,121}$ Toothed rotor-stator mixers (Fig. 7) are used as wet mills for nucleation, with multiple tooth configurations (coarse, medium, fine, multi-stage). ${ }^{57,122}$ Compared to impinging jet micromixers which are easy to implement in continuous flow, the rotor-stator mixers have industrial-scale equipment readily available, and do not need to balance the momentum of two streams. ${ }^{121}$ For high primary nucleation, a small turnover number per residence time (defines how frequently the slurry in MSMPR is milled) within the wet mill is preferred (e.g., no recycle). ${ }^{57}$ Other design parameters of wet milling are discussed in post-addition seed treatment subsection later.

\subsection{Seed addition}

Seed crystals can be added discretely or continuously during the continuous crystallization process. Seed crystals are typically added under solution conditions where the nucleation rate is low. ${ }^{4,47,123}$

(1) Discrete seed addition

Discrete seed addition occurs after off-line seed generation. Usually off-line seeds are added at the start-up of (and sometimes also periodically during ${ }^{49}$ ) the continuous process, then secondary nucleation will continue to provide the seeds to keep the process going. These seeds come from a previous process as discussed in the off-line seeding subsection earlier. This addition mode is adopted by most MSMPRs, fluidized bed crystallizers, ${ }^{62,69}$ and some other crystallizers. The seed load, size, and timing of these off-line seeds may follow the same design criteria $^{5,16}$ for batch crystallizers, with consideration of the process difference, such as on residence time and clogging. Due to the equipment similarity, MSMPRs can have very similar seeding addition location as in batch crystallizers, and can use similar seeding strategies during their startup..$^{52,66}$

(2) Continuous seed addition

Continuous seed addition occurs after seed generation, for both off-line and in situ seed generation. Almost all continuous crystallizers can adopt continuous seed addition mode, including some discussed in the discrete addition mode. While most MSMPRs adopt off-line seeds (e.g., at the first tank), downstream tanks of these multi-stage MSMPRs adopt continuous seed addition at the same time. A few segmented flow crystallizers have adopted off-line seeds, while the majority used seeds generated in real time, for most segmented/slug flow crystallizers ${ }^{10,14,29}$ and micromixer crystallizers. Regardless of which continuous crystallizer configuration, the addition of seed slurry can use the same equipment (e.g., pump and tubing) in the same way as in the addition of solution. No additional equipment is needed, and all equip- ment associated with the in situ nucleation method described in the subsection 5.1 applies here. For example, tubular crystallizers receive seeds from flow along the tubing (e.g., through $\mathrm{T}$ or $\mathrm{Y}$ mixers), and downstream MSMPR tanks receive seeds from upstream tanks through their regular tankto-tank transfer lines. Note that sometimes the total mass of seeds and number density from in situ methods can be difficult to control due to the tiny mass of nuclei, but usually the main process consideration is to minimize the potential for clogging.

\subsection{Post-addition seed treatment}

Seed treatment is optional, and this post-nucleation process can be easier if nucleation is already well controlled.

(1) Off-line treatment (pre-addition)

Off-line seed treatment occurs after off-line seed generation and before off-line seed addition. All existing methods developed for batch crystallization can be used to tune seed crystal size off-line. For example, milling, ${ }^{46,121,124-126}$ sonication, and heating the slurry ${ }^{38}$ can be used to reduce crystal size or improve uniformity. These methods are also useful for in situ mode. Sieving ${ }^{46}$ or filtration can be used to select seed crystals of a desired size range.

(2) In-line treatment (post addition)

In-line seed treatment occurs after in-line seed addition (for both modes of seed generation), to continuously provide small seed crystals. Recycling small seeds back to the main crystallizer for further growth has been effective in keeping growth rate high while controlling product crystal qualities. High shear wet milling has often been used to reduce average crystal size downstream of MSMPR crystallizers, mainly through mass fracture, attrition, and secondary nucleation. ${ }^{54-57,121,125,126}$ Compared to the industrial tradition of reducing particle size using dry milling (e.g., pin, jet), wet milling can avoid many operational issues (e.g., stability, dusting), and improves process integration by combining with crystallization unit operation. ${ }^{54-57,121}$ Most parameters of rotor stator wet mills have been evaluated for batches. Generally speaking, the larger the energy output (e.g., normalization through an energy factor and a probability factor), or the finer the tooth geometry (e.g., fine vs. coarse), the smaller the particle size. ${ }^{57,126,127}$ For a defined mill head (tooth) geometry, larger tip (rotational) speed leads to larger recycling flow rate (and cycle number, or turnover number per residence time), and usually more secondary nucleation and crystal breakage (smaller average crystal size). ${ }^{55,126} \mathrm{Be}-$ sides high shear wet milling, wet media milling (using milling beads) has also been used in continuous recirculation mode for reducing drug particle size in suspensions to nanoparticle scale (details described elsewhere ${ }^{128,129}$ ). Similarly, ultrasonication has been used in fluidized bed crystallizers to generate smaller seed crystals by breaking larger crystals and secondary nucleation, ${ }^{33,46,62,69}$ or used in tubular crystallizers to reduce wall encrustation. ${ }^{12}$ These seeds have more surface area for growth per unit volume compared with crystals 
before breakage. Both methods (wet milling and ultrasonication) have been described in detail earlier in this section on in-line nucleation.

Temperature cycling (sometimes referred to as fines dissolution) can selectively remove tiny crystals of those compounds with temperature-dependent solubility. ${ }^{10,29,33,104,130}$ The $\mathrm{T}$ cycling method can be used to narrow the CSD in various tubular crystallizers and in the recycle line of MSMPRs, ${ }^{10,131}$ and to reduce fouling from tubular crystallizers (e.g., with feedback control on temperature $\left.{ }^{131}\right)$. The cycling method can also be used together with other in-line methods discussed earlier, such as micromixers ${ }^{10}$ or wet milling. ${ }^{125}$ A typical $\mathrm{T}$ cycle is composed of the heating dissolution and the cooling growth (recrystallization) sub-processes. From total solute mass balance, each existing crystal (especially the larger crystals) will become larger after temperature cycles, as the total number of crystals is reduced in the heating sub-process (fines removal). ${ }^{38}$ The temperature zones are usually generated by convection (e.g., water) or conduction (e.g., metal), such as jackets for MSMPRs (e.g., Fig. 2a and b), or heat exchangers ${ }^{132}$ (e.g., Fig. 3a and b) and circulation baths ${ }^{10}$ (e.g., Fig. 4b and c) for tubing (tubular crystallizer, or the recycle line of MSMPRs). Usually heating can be fast but cooling needs to be slower, to dissolve smaller crystals and grow larger crystals without secondary nucleation. ${ }^{10,131}$ The total number of cycles can be determined by both the final CSD requirement and the size dependence of growth and dissolution kinetics. ${ }^{104}$ For example, multiple cycles are preferred to a single cycle if such size dependence exists. $^{104}$

Besides reducing the size of large crystals as discussed earlier, the small seed crystals can also be selected in real time from the product crystal slurry to recycle back into the original crystallizer for higher yield and faster kinetics. ${ }^{68}$ There are two common in-line recycling selection methods: crystal filtration and gravity sedimentation, ${ }^{63}$ both mainly used for MSMPR crystallizers. The in-line filtration method has been also used in fluidized bed crystallization for seeding, ${ }^{69}$ and in slug flow crystallization for carrier fluid recycling ${ }^{30}$ as well. The selection/separation mechanism for filtration is crystal size. The in-line filtration method allows crystals with sizes smaller than the filter pore size to pass through and be recycled, while larger crystals are retained by the filter. As with off-line filtration, the common equipment includes membrane or glass filters, or filter paper (Fig. 2c). The crystals that are selected depend on the filter properties, such as molecular weight cut-off, hydrophobicity, and polymer type. ${ }^{59}$ The selection/separation mechanism for sedimentation is centrifugal force or gravity. One sedimentation method separates crystals from clear mother liquor in a column separator (Fig. 2d), before recycling crystals back to the MSMPR crystallizers. This approach not only enhanced the yield with reduced residence time, but also maintained the crystal purity. ${ }^{63}$ As the recycling selection method is part of the recycle loop, the recycle ratio ${ }^{63,64}$ and flow rate for the recycle stream are also important parameters.

\section{A case study: design of a segmented/slug flow continuous cooling crystallizer with in-line fo- cused nucleation}

Steps 1 and 2: setting goals and initial batch experiments

Consider the goal of designing a slug-flow continuous crystallization process that can economically and efficiently generate large uniform crystals with reduced fouling. L-Asparagine monohydrate (LAM) is the model compound, ${ }^{46,105,107,133}$ due to its representative solubility and operational difficulty in generating uniform-size crystals. ${ }^{5,15}$ Deionized water was selected as a green solvent that can produce a reasonable yield $\left(\sim 85 \%\right.$ from $70{ }^{\circ} \mathrm{C}$ to $\left.25{ }^{\circ} \mathrm{C}\right)$ for cooling crystallization, ${ }^{123}$ and only one crystalline form has been observed under these conditions.

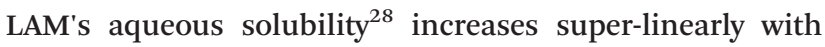
temperature increase, very similar to many pharmaceuticals in good solvents. An accurate solubility curve was measured in a batch crystallizer, based on the absorbance spectra from attenuated total reflectance-Fourier transform infrared (ATRFTIR) spectroscopy and chemometrics calibration model. ${ }^{47}$ The starting and final temperatures for cooling crystallization were also set based on the solubility curve and operational considerations. For example, the final (low) temperature was set as the ambient temperature, as the LAM solubility in aqueous solution is low (e.g., $\sim 3 \mathrm{wt} \%$ ), and only a moderate increase in yield would result from lowering the temperature further. The starting (high) temperature was set at $70{ }^{\circ} \mathrm{C}$ to achieve high yield, which is also low enough that LAM has insignificant chemical degradation. The yield could be increased by increasing the starting cooling and/or by combining temperature with anti-solvent crystallization. . $^{5,16,104}$

The rough time scale for the crystal growth process was estimated in sub-liter-scale batch crystallizations. For example, a seeded batch crystallization in a stirred tank was completed within one hour, even at low relative supersaturation (e.g., 1.05 to 1.15 ) and non-optimized seed conditions. Potential difficulty in operation was also observed, which was the tendency to aggregate, and secondary nucleation at higher supersaturation. $^{47}$

Steps 3 and 4: selecting the operational mode and designing the crystallizer configuration

The continuous operational mode and the slug flow crystallizer configuration are set by the project goal. Fouling or clogging is a typical issue to prevent and/or address in a tubularflow crystallizer. The slug-flow crystallizer (Fig. 4a-d) reduces fouling by exploiting multi-phase hydrodynamics and recirculation inside the slurry slugs to push solids away from tubing wall into the tubing center (Fig. 4d). Operational convenience is also a consideration, due to the tendency of LAM towards aggregation, which increases the potential for crystallizer fouling and clogging. In slug flow crystallizers, no 
stirring blades are needed, which minimizes the possibility of crystal breakage which would generate tiny particles prone towards aggregation. Additional design or configuration options are also available for slug flow crystallizers to reduce fouling, such as by using carrier fluid (replacing air) to separate liquid slugs, followed by recovery holes, or by including a split "mouth" design to induce turbulence. ${ }^{30}$

For scaling up, most continuous processes need additional experiments and troubleshooting in larger scale equipment (e.g., larger diameter tubing). For slug-flow tubular crystallizers, the flow pattern remains the same for larger production rates. The scale-up of production rate is to increase the number of slugs with exactly the same equipment and process, by using a longer tube rather than by increasing the tube diameter. In this way, laboratory-scale experiments may directly indicate manufacturing-scale performance. The equipment needs are simple, which are standard peristaltic pumps and disposable tubing.

\section{Step 5: improved control of crystal size with process design and seeding strategy}

Each slug is like an individual batch crystallizer moving in the tube, so all previous knowledge and experience for batch control and optimization can be applied. ${ }^{14,132}$ Similar as many other continuous crystallizers, the crystallization design in Fig. 4 adopts the strategy of decoupling nucleation and growth for enhanced individual control. The decoupling is achieved by focused in situ nucleation before slug formation, together with controlled growth of these nuclei in individual slugs under different conditions than the nucleation stage. These nuclei serve as seeds in the slugs, the same way as seeds in batches. Then these slugs go through temperaturevarying regions so that the dissolution and/or growth of crystals inside the slugs are controlled. The spatial temperature profile along tubing that each slug goes through within the residence time, is the same way that a batch crystallizer goes through a time-variant temperature profile within the residence time.

Various in situ seeding methods were evaluated. Direct cooling of hot LAM aqueous solution moving in tubing without slugs results in aggregation and crystal size variation. ${ }^{10}$ Such phenomena of aggregation and clogging are typical, usually indicating too high supersaturation. Then nucleation methods were evaluated that maintain a localized region with high supersaturation (for nucleation) while reducing the overall supersaturation. ${ }^{28}$ Micromixers and sonication are such two methods, reviewed in section 4 , that can avoid clogging when connected to a slug-flow crystallization process: ${ }^{10,14}$

(1) Focused micromixer nucleation is achieved by combining hot and cold saturated solutions through micromixers. The average supersaturation of the two streams is low, while the high supersaturation region is located on the hot stream side, and close to the hot-cold stream interface. Experiments using various configurations of such micromixers result in crystals of improved size uniformity and reduced aggregation
(Fig. 5c). ${ }^{10}$ Among common configurations, the dualimpinging jet (DIJ, Fig. 5) has higher mixing intensity due to nonzero jet angles; whereas the coaxial mixer with parallel streams (e.g., Fig. 4c) has lower clogging probability, as high supersaturation occurs near the tube center and far from the wall where the velocity is low. The flow rates are chosen based on consideration of both the production rate (and waste production rate) and mixing intensity. ${ }^{7,105}$

(2) Focused nucleation aided with sonication is achieved by placing the sonication probe against the outer wall of tubing under temperature control (Fig. 6). Inside the tube filled with solution, the highest sonication intensity is received right under the probe tip, ${ }^{118}$ and the intensity rapidly decays even a small distance upstream and downstream. This design narrows the spatial variation on sonication intensity by localizing the nucleation zone to only a small portion of the tubing (the sonication zone marked in Fig. 6). The sonication increases the nucleation rate even at a smaller degree of cooling (supersaturation), which is usually insufficient for nucleation without sonication. Additional tuning capability is gained by additional degree of freedom from sonication such as the applied power. The power was chosen to produce a strong enough excitation to promote nucleation while not too high to increase the localized temperature (which would dissolve some nuclei). ${ }^{14}$ In this way, nucleation rate can be varied without changing the production rate (flow rate). ${ }^{14}$

Post-nucleation treatment is applied when the average size of product crystals needs to be increased, and/or the amount of fine crystals to be reduced. ${ }^{10}$ For this compound (LAM) with temperature-dependent solubility, spatially varying temperature profiles along the tube can be applied for controlled dissolution and growth processes. The $\mathrm{T}$ profile is also specified (e.g., in Fig. 4b-d) so that no sudden drop in temperature occurs which could generate too many solids in a short time. The post-nucleation treatment can be easier when the nuclei is under good control. For example, when sonicationaided focused nucleation was used, even natural cooling through tubing wall (usually not an optimized cooling profile) was sufficient during the growth stage. ${ }^{14}$ The experimental observations indicated minimal aggregation and no clogging, with tunable crystal sizes (e.g., Fig. 4d). ${ }^{134}$

\section{Outlook and future perspectives}

Continuous crystallization has received increased attention for improving the consistency of product quality and the efficiency of manufacturing processes. The design procedure (section 2) and specific configuration (section 3) for continuous crystallizers may evolve based on possibly changing needs: (1) process requirements will be higher and with more constraints; (2) more accurate thermodynamic and kinetic data will be collected in shorter time (e.g., high-throughput screening); (3) more varieties of crystallizer configurations will be available and improved; and (4) detailed design for any selected crystallizer configuration may be achieved by software to accelerate the exploration of novel designs. For 
process requirements specifically, future continuous crystallizers are expected to retain the strengths of the current continuous crystallizer designs, ${ }^{14}$ and to achieve simultaneous control over multiple crystal properties (e.g., polymorphic identity and morphology), while removing or suppressing the weaknesses. At the same time, scale-up and operational problems need to be minimized, such as process disturbances and variations in crystallization kinetics (e.g., due to changes in the contaminant profile in the feed streams), time duration and materials wasted during startup, and fouling/ clogging.

These design improvements may benefit from advanced process monitoring and control tools ${ }^{3,47,54,56,131,135}$ (with suitable in-line process analytical technology ${ }^{32,58,136}$ ) and predictive models ${ }^{6,49,58,60,61,67,68,133,137,138}$ (based on deeper mechanistic understanding of the physical chemical phenomena). Process intensification strategies from existing crystallizers $^{1-11,139}$ (batch or continuous) can also be helpful for future improvement, including: (1) decoupling nucleation and growth; (2) exploiting multiphase hydrodynamics for enhanced mixing (Table 1 and section 3), e.g., oscillatory flow, Taylor flow, or slug flow with recirculation; ${ }^{10}$ and (3) enhancing seeding control (section 4) and growth control (section 5), e.g., control crystal size in flow with wet milling, ultrasonication, micromixers, temperature cycling (fines removal), ${ }^{10,29}$ and recycling method selection. Further development and additional methods are also expected for advanced nucleation control.

\section{Conflicts of interest}

There are no conflicts of interest to declare.

\section{References}

1 A. S. Myerson, M. Krumme, M. Nasr, H. Thomas and R. D. Braatz, J. Pharm. Sci., 2015, 104, 832-839.

2 L. X. Yu, Pharm. Res., 2008, 25, 781-791.

3 Z. K. Nagy and R. D. Braatz, Annu. Rev. Chem. Biomol. Eng., 2012, 3, 55-75.

4 J. W. Mullin, Crystallization, Butterworth-Heinemann, Oxford, 4th edn, 2001.

5 A. S. Myerson, Handbook of Industrial Crystallization, Butterworth-Heinemann, Woburn, MA, 2nd edn, 2002.

6 H. Zhang, J. Quon, A. J. Alvarez, J. Evans, A. S. Myerson and B. Trout, Org. Process Res. Dev., 2012, 16, 915-924.

7 M. Jiang, Y.-E. Li, H.-H. Tung and R. D. Braatz, Chem. Eng. Process., 2015, 97, 242-247.

8 H.-H. Tung, Org. Process Res. Dev., 2013, 17, 445-454.

9 R. J. P. Eder, S. Radl, E. Schmitt, S. Innerhofer, M. Maier, H. Gruber-Woelfler and J. G. Khinast, Cryst. Growth Des., 2010, 10, 2247-2257.

10 M. Jiang, Z. Zhu, E. Jimenez, C. D. Papageorgiou, J. Waetzig, A. Hardy, M. Langston and R. D. Braatz, Cryst. Growth Des., 2014, 14, 851-860.
11 E. Drioli, G. Di Profio and E. Curcio, Membrane-Assisted Crystallization Technology, Imperial College Press, London, U.K., 2015.

12 O. Narducci, A. G. Jones and E. Kougoulos, Chem. Eng. Sci., 2011, 66, 1069-1076.

13 R. Jamshidi, D. Rossi, N. Saffari, A. Gavriilidis and L. Mazzei, Cryst. Growth Des., 2016, 16, 4607-4619.

14 M. Jiang, C. D. Papageorgiou, J. Waetzig, A. Hardy, M. Langston and R. D. Braatz, Cryst. Growth Des., 2015, 15, 2486-2492.

15 H.-H. Tung, E. L. Paul, M. Midler and J. A. McCauley, Crystallization of Pharmaceuticals: An Industrial Perspective, Wiley, Hoboken, NJ, 2009.

16 E. A. Lewis, M. M. Seckler, H. Kramer and G. van Rosmalen, Industrial crystallization: Fundamentals and applications, Cambridge University Press, Cambridge, 2015.

17 R. Vacassy, J. Lemaître, H. Hofmann and J. H. Gerlings, AIChE J., 2000, 46, 1241-1252.

18 N. E. B. Briggs, U. Schacht, V. Raval, T. McGlone, J. Sefcik and A. J. Florence, Org. Process Res. Dev., 2015, 19, 1903-1911.

19 C. X. Zhao, L. He, S. Z. Qiao and A. P. J. Middelberg, Chem. Eng. Sci., 2011, 66, 1463-1479.

20 P. Moschou, M. H. J. M. De Croon, J. Van Der Schaaf and J. C. Schouten, Rev. Chem. Eng., 2014, 30, 127-138.

21 K. Robertson, Chem. Cent. J., 2017, 11, 4.

22 J. M. Köhler, S. Li and A. Knauer, Chem. Eng. Technol., 2013, 36, 887-899.

23 G. H. Albuquerque and G. S. Herman, Cryst. Growth Des., 2017, 17, 156-162.

24 K. J. Kim, R. P. Oleksak, E. B. Hostetler, D. A. Peterson, P. Chandran, D. M. Schut, B. K. Paul, G. S. Herman and C. H. Chang, Cryst. Growth Des., 2014, 14, 5349-5355.

25 L. Yu, Y. Pan, C. Wang and L. Zhang, Chem. Eng. J., 2013, 219, 78-85.

26 A. M. Nightingale and J. C. Demello, Adv. Mater., 2013, 25, 1813-1821.

27 N. Jongen, M. Donnet, P. Bowen, J. Lemaître, H. Hofmann, R. Schenk, C. Hofmann, M. Aoun-Habbache, S. GuillemetFritsch, J. Sarrias, A. Rousset, M. Viviani, M. T. Buscaglia, V. Buscaglia, P. Nanni, A. Testino and R. Herguijuela, Chem. Eng. Technol., 2003, 26, 303-305.

28 M. Jiang, C. Gu and R. D. Braatz, Chem. Eng. Process., 2015, 97, 187-194.

29 R. J. P. Eder, S. Schrank, M. O. Besenhard, E. Roblegg, H. Gruber-Woelfler and J. G. Khinast, Cryst. Growth Des., 2012, 12, 4733-4738.

30 K. Robertson, P. B. Flandrin, A. R. Klapwijk and C. C. Wilson, Cryst. Growth Des., 2016, 16, 4759-4764.

31 N. Variankaval, A. S. Cote and M. F. Doherty, AIChE J., 2008, 54, 1682-1688.

32 Z. Gao, S. Rohani, J. Gong and J. Wang, Engineering, 2017, 3, 343-353.

33 S. Kim, C. Wei and S. Kiang, Org. Process Res. Dev., 2003, 7, 997-1001.

34 C. C. Sun, Expert Opin. Drug Delivery, 2013, 10, 201-213. 
35 Polymorphism in the pharmaceutical industry, ed. R. Hilfiker, Wiley-VCH, Weinheim, 2006.

36 S. M. Miller and J. B. Rawlings, AIChE J., 1994, 40, 1312-1327.

37 T. Togkalidou, H.-H. Tung, Y. Sun, A. T. Andrews and R. D. Braatz, Ind. Eng. Chem. Res., 2004, 43, 6168-6181.

38 M. Jiang, X. Zhu, M. C. Molaro, M. L. Rasche, H. Zhang, K. Chadwick, D. M. Raimondo, K.-K. K. Kim, L. Zhou, Z. Zhu, M. H. Wong, D. O'Grady, D. Hebrault, J. Tedesco and R. D. Braatz, Ind. Eng. Chem. Res., 2014, 53, 5325-5336.

39 Chemical Engineering in the Pharmaceutical Industry: $R \& D$ to Manufacturing, Wiley, Hoboken, N.J., ed. D. J. am Ende, 2010.

40 S. Lawton, G. Steele, P. Shering, L. Zhao, I. Laird and X. Ni, Org. Process Res. Dev., 2009, 13, 1357-1363.

41 A. J. Alvarez and A. S. Myerson, Cryst. Growth Des., 2010, 10, 2219-2228.

42 M. Midler, E. L. Paul, E. F. Whittington, M. Futran, P. D. Liu, J. Hsu and S. H. Pan, Crystallization method to improve crystal structure and size, U.S. Pat., 5314506A, 1994.

43 T. McGlone, N. E. B. Briggs, C. A. Clark, C. J. Brown, J. Sefcik and A. J. Florence, Org. Process Res. Dev., 2015, 19, 1186-1202.

44 S. R. L. Gobert, S. Kuhn, L. Braeken and L. C. J. Thomassen, Org. Process Res. Dev., 2017, 21, 531-542.

45 M. Midler, Process for production of crystals in fluidized bed crystallizers, U.S. Pat., 3892539A, 1975.

46 D. Binev, A. Seidel-Morgenstern and H. Lorenz, Chem. Eng. Sci., 2015, 133, 116-124.

47 M. Jiang, M. H. Wong, Z. Zhu, J. Zhang, L. Zhou, K. Wang, A. N. Ford Versypt, T. Si, L. M. Hasenberg, Y. E. Li and R. D. Braatz, Chem. Eng. Sci., 2012, 77, 2-9.

48 H. Lorenz and A. Seidel-Morgenstern, Angew. Chem., Int. Ed., 2014, 53, 1218-1250.

49 S. Qamar, M. Peter Elsner, I. Hussain and A. SeidelMorgenstern, Chem. Eng. Sci., 2012, 71, 5-17.

50 A. J. Alvarez, A. Singh and A. S. Myerson, Cryst. Growth Des., 2011, 11, 4392-4400.

51 Q. Su, C. D. Rielly, K. A. Powell and Z. K. Nagy, AIChE J., 2017, 63, 1313-1327.

52 Y. Yang and Z. K. Nagy, Ind. Eng. Chem. Res., 2015, 54, 5673-5682.

53 R. Peña and Z. K. Nagy, Cryst. Growth Des., 2015, 15, 4225-4236.

54 Y. Yang, L. Song, Y. Zhang and Z. K. Nagy, Ind. Eng. Chem. Res., 2016, 55, 4987-4996.

55 Y. Yang, L. Song, T. Gao and Z. K. Nagy, Cryst. Growth Des., 2015, 15, 5879-5885.

56 Y. Yang and Z. K. Nagy, Chem. Eng. Sci., 2015, 127, 362-373.

57 Y. Yang, L. Song and Z. K. Nagy, Cryst. Growth Des., 2015, 15, 5839-5848.

58 K. A. Powell, A. N. Saleemi, C. D. Rielly and Z. K. Nagy, Chem. Eng. Process., 2015, 97, 195-212.
59 S. Ferguson, F. Ortner, J. Quon, L. Peeva, A. Livingston, B. L. Trout and A. S. Myerson, Cryst. Growth Des., 2014, 14, 617-627.

60 G. Power, G. Hou, V. K. Kamaraju, G. Morris, Y. Zhao and B. Glennon, Chem. Eng. Sci., 2015, 133, 125-139.

61 D. Zhang, S. Xu, S. Du, J. Wang and J. Gong, Engineering, 2017, 3, 354-364.

62 C. Rougeot and J. E. Hein, Org. Process Res. Dev., 2015, 19, 1809-1819.

63 J. Li, B. L. Trout and A. S. Myerson, Org. Process Res. Dev., 2016, 20, 510-516.

64 S. Y. Wong, A. P. Tatusko, B. L. Trout and A. S. Myerson, Cryst. Growth Des., 2012, 12, 5701-5707.

65 T. C. Lai, J. Cornevin, S. Ferguson, N. Li, B. L. Trout and A. S. Myerson, Cryst. Growth Des., 2015, 15, 3374-3382.

66 G. Hou, G. Power, M. Barrett, B. Glennon, G. Morris and Y. Zhao, Cryst. Growth Des., 2014, 14, 1782-1793.

67 A. D. Randolph and M. A. Larson, Theory of Particulate Processes: Analysis and Techniques of Continuous Crystallization, Academic Press, New York, 2nd edn, 1974.

68 B. Wood, K. P. Girard, C. S. Polster and D. M. Croker, Org. Process Res. Dev., 2019, 23, 122-144.

69 D. Binev, A. Seidel-Morgenstern and H. Lorenz, Cryst. Growth Des., 2016, 16, 1409-1419.

70 H. Siddique, C. J. Brown, I. Houson and A. J. Florence, Org. Process Res. Dev., 2015, 19, 1871-1881.

71 P. Cruz, F. Rocha and A. Ferreira, CrystEngComm, 2016, 18, 9113-9121.

72 C. J. Brown, J. A. Adelakun and X. Ni, Chem. Eng. Process., 2015, 97, 180-186.

73 X. Ni, M. R. Mackley, A. P. Harvey, P. Stonestreet, M. H. I. Baird and N. V. Rama Rao, Chem. Eng. Res. Des., 2003, 81, 373-383.

74 J. R. McDonough, A. N. Phan and A. P. Harvey, Chem. Eng. J., 2015, 265, 110-121.

75 W. M. Deen, Analysis of Transport Phenomena, Oxford University Press, New York, 2nd edn, 2012.

76 J. R. Méndez del Río and R. W. Rousseau, Cryst. Growth Des., 2006, 6, 1407-1414.

77 S. Ferguson, G. Morris, H. Hao, M. Barrett and B. Glennon, Chem. Eng. Sci., 2012, 77, 105-111.

78 M. O. Besenhard, P. Neugebauer, C. Da-Ho and J. G. Khinast, Cryst. Growth Des., 2015, 15, 1683-1691.

79 L. Hohmann, R. Gorny, O. Klaas, J. Ahlert, K. Wohlgemuth and N. Kockmann, Chem. Eng. Technol., 2016, 39, 1268-1280.

80 M. Raphael, Can. J. Chem. Eng., 1995, 77, 470-483.

81 C. Brechtelsbauer and F. Ricard, Org. Process Res. Dev., 2001, 5, 646-651.

82 A. T. Nguyen, J. M. Kim, S. M. Chang and W. S. Kim, Ind. Eng. Chem. Res., 2011, 50, 3483-3493.

83 A. T. Nguyen, T. Yu and W. S. Kim, J. Cryst. Growth, 2017, 469, 65-77.

84 X. Zhou, X. Zhu, B. Wang, J. Li, Q. Liu, X. Gao, K. K. Sirkar and D. Chen, J. Membr. Sci., 2018, 564, 682-690. 
85 J. C. W. Fern, S. Ohsaki, S. Watano and R. Pfeffer, Int. J. Pharm., 2018, 543, 139-150.

86 D. Chen, D. Singh, K. K. Sirkar and R. Pfeffer, Langmuir, 2015, 31, 432-441.

87 E. Chabanon, D. Mangin and C. Charcosset, J. Membr. Sci., 2016, 509, 57-67.

88 M. Furuta, K. Mukai, D. Cork and K. Mae, Chem. Eng. Process., 2016, 102, 210-218.

89 B. Rimez, R. Debuysschère, J. Conté, E. Lecomte-Norrant, C. Gourdon, P. Cognet and B. Scheid, Cryst. Growth Des., 2018, 18, 6431-6439.

90 V. Wiedmeyer, F. Anker, C. Bartsch, A. Voigt, V. John and K. Sundmacher, Ind. Eng. Chem. Res., 2017, 56, 3699-3712.

91 L. Hohmann, S. K. Kurt, S. Soboll and N. Kockmann, J. Flow Chem., 2016, 6, 181-190.

92 L. Hohmann, T. Greinert, O. Mierka, S. Turek, G. Schembecker, E. Bayraktar, K. Wohlgemuth and N. Kockmann, Cryst. Growth Des., 2018, 18, 1459-1473.

93 P. Neugebauer and J. G. Khinast, Cryst. Growth Des., 2015, 15, 1089-1095.

94 S. Guillemet-Fritsch, M. Aoun-Habbache, J. Sarrias, A. Rousset, N. Jongen, M. Donnet, P. Bowen and J. Lemaître, Solid State Ionics, 2004, 171, 135-140.

95 T. Yonemoto, M. Kubo, T. Doi and T. Tadaki, Chem. Eng. Res. Des., 1997, 75, 413-419.

96 A. Testino, F. Pilger, M. A. Lucchini, J. E. Q. Quinsaat, C. Stähli and P. Bowen, Molecules, 2015, 20, 10566-10581.

97 M. Su and Y. Gao, Ind. Eng. Chem. Res., 2018, 57, 3781-3791.

98 C. Daniel Scott, R. Labes, M. Depardieu, C. Battilocchio, M. G. Davidson, S. V. Ley, C. C. Wilson and K. Robertson, React. Chem. Eng., 2018, 3, 631-634.

99 J. Lu, J. D. Litster and Z. K. Nagy, Cryst. Growth Des., 2015, 15, 3645-3651.

100 S. Kudo and H. Takiyama, J. Chem. Eng. Jpn., 2012, 45, 305-309.

101 J. Schiewe and B. Zierenberg, Process and apparatus for producing inhalable medicaments, U.S. Pat., 20030015194A1, 2003.

102 J. Lemaître, N. Jongen, R. Vacassy and P. Bowen, Production of Powders, U.S. Pat., 6458335B1, 2002.

103 J. H. Chaaban, K. Dam-Johansen, T. Skovby and S. Kiil, Org. Process Res. Dev., 2013, 17, 1010-1020.

104 M. O. Besenhard, P. Neugebauer, O. Scheibelhofer and J. G. Khinast, Cryst. Growth Des., 2017, 17, 6432-6444.

105 A. J. Mahajan and D. J. Kirwan, AIChE J., 1996, 42, 1801-1814.

106 H. Wang, A. Mustaffar, A. N. Phan, V. Zivkovic, D. Reay, R. Law and K. Boodhoo, Chem. Eng. Process., 2017, 118, 78-107.

107 C. Lindenberg and M. Mazzotti, AIChE J., 2011, 57, 942-950.

108 B. K. Johnson and R. K. Prud'homme, AIChE J., 2003, 49, 2264-2282.

109 C. Lindenberg and M. Mazzotti, Chem. Eng. Sci., 2009, 64, 4286-4294.
110 D. L. Marchisio, L. Rivautella and A. A. Barresi, AIChE J., 2006, 52, 1877-1887.

111 Z. Gao, F. Altimimi, J. Gong, Y. Bao, J. Wang and S. Rohani, Cryst. Growth Des., 2018, 18, 2628-2635.

112 T. Nguyen, A. Khan, L. Bruce, C. Forbes, R. O'Leary and C. Price, Crystals, 2017, 7, 294.

113 H. Kim and K. Suslick, Crystals, 2018, 8, 280.

114 J. R. G. Sander, B. W. Zeiger and K. S. Suslick, Ultrason. Sonochem., 2014, 21, 1908-1915.

115 H. N. Kim, J. R. G. Sander, B. W. Zeiger and K. S. Suslick, Cryst. Growth Des., 2015, 15, 1564-1567.

116 D. Fernandez Rivas and S. Kuhn, Top. Curr. Chem., 2016, 374, 70.

117 H. M. Santos and J. L. Capelo, Talanta, 2007, 73, 795-802.

118 M. Jiang, C. Gu and R. D. Braatz, Chem. Eng. Process., 2017, 117, 186-194.

119 E. Miyasaka, Y. Kato, M. Hagisawa and I. Hirasawa, J. Cryst. Growth, 2006, 289, 324-330.

120 S. Gracin, M. Uusi-Penttilä and Å. C. Rasmuson, Cryst. Growth Des., 2005, 5, 1787-1794.

121 T. Kamahara, M. Takasuga, H. H. Tung, K. Hanaki, T. Fukunaka, B. Izzo, J. Nakada, Y. Yabuki and Y. Kato, Org. Process Res. Dev., 2007, 11, 699-703.

122 B. Ahmed, C. J. Brown, T. McGlone, D. L. Bowering, J. Sefcik and A. J. Florence, Int. J. Pharm., 2019, 554, 201-211.

123 C. J. Brown, T. McGlone, S. Yerdelen, V. Srirambhatla, F. Mabbott, R. Gurung, M. L. Briuglia, B. Ahmed, H. Polyzois, J. McGinty, F. Perciballi, D. Fysikopoulos, P. MacFhionnghaile, H. Siddique, V. Raval, T. S. Harrington, A. D. Vassileiou, M. Robertson, E. Prasad, A. Johnston, B. Johnston, A. Nordon, J. S. Srai, G. Halbert, J. H. ter Horst, C. J. Price, C. D. Rielly, J. Sefcik and A. J. Florence, Mol. Syst. Des. Eng., 2018, 3, 518-549.

124 A. Harter, L. Schenck, I. Lee and A. Cote, Org. Process Res. Dev., 2013, 17, 1335-1344.

125 E. Kougoulos, I. Smales and H. M. Verrier, AAPS PharmSciTech, 2011, 12, 287-294.

126 J. Engstrom, C. Wang, C. Lai and J. Sweeney, Int. J. Pharm., 2013, 456, 261-268.

127 C. V. Luciani, E. W. Conder and K. D. Seibert, Org. Process Res. Dev., 2015, 19, 582-589.

128 A. Monteiro, A. Afolabi and E. Bilgili, Drug Dev. Ind. Pharm., 2013, 39, 266-283.

129 M. Malamatari, K. M. G. Taylor, S. Malamataris, D. Douroumis and K. Kachrimanis, Drug Discovery Today, 2018, 23, 534-547.

130 Z. Wu, S. Yang and W. Wu, CrystEngComm, 2016, 18, 2222-2238.

131 A. Koswara and Z. K. Nagy, in IFAC-PapersOnLine, 2015, vol. 28, pp. 193-198.

132 M. L. Rasche, M. Jiang and R. D. Braatz, Comput. Chem. Eng., 2016, 95, 240-248.

133 T. Vetter, C. L. Burcham and M. F. Doherty, Chem. Eng. Sci,, 2014, 106, 167-180. 
134 M. Jiang and R. D. Braatz, Chem. Eng. Technol., 2018, 41, 143-148.

135 C. Darmali, S. Mansouri, N. Yazdanpanah and M. W. Woo, Ind. Eng. Chem. Res., 2019, 58, 1463-1479.

136 L. L. Simon, H. Pataki, G. Marosi, F. Meemken, K. Hungerbuhler, A. Baiker, S. Tummala, B. Glennon, M. Kuentz, G. Steele, H. J. M. Kramer, J. W. Rydzak, Z. Chen, J. Morris, F. Kjell, R. Singh, R. Gani, K. V. Gernaey, M. Louhi-Kultanen, J. Oreilly, N. Sandler, O. Antikainen, J. Yliruusi, P. Frohberg, J. Ulrich, R. D. Braatz, T. Leyssens, M. Von Stosch, R. Oliveira, R. B. H. Tan, H. Wu, M. Khan, D. Ogrady, A. Pandey, R. Westra, E. DelleCase, D. Pape, D. Angelosante, Y. Maret, O. Steiger, M.
Lenner, K. Abbou-Oucherif, Z. K. Nagy, J. D. Litster, V. K. Kamaraju and M. Sen Chiu, Org. Process Res. Dev., 2015, 19, 3-62.

137 T. Wang, H. Lu, J. Wang, Y. Xiao, Y. Zhou, Y. Bao and H. Hao, J. Ind. Eng. Chem., 2017, 54, 14-29.

138 D. W. Griffin, D. A. Mellichamp and M. F. Doherty, Chem. Eng. Sci., 2010, 65, 5770-5780.

139 J. Wang, F. Li and R. Lakerveld, Chem. Eng. Process., 2018, 127, 111-126.

140 S. V. Dalvi and R. N. Dave, Ind. Eng. Chem. Res., 2009, 48, 7581-7593.

141 S. Devarakonda, J. M. B. Evans and A. S. Myerson, Cryst. Growth Des., 2004, 4, 687-690. 